\title{
EXPLORING URBAN ACCESSIBILITY SCORES USING MULTI-CRITERIA DECISION ANALYSIS TECHNIQUES
}

\author{
by \\ Anna Racovali \\ Western University, 2016 \\ A major research paper \\ presented to Ryerson University \\ in partial fulfillment of the \\ requirements for the degree of \\ Master of Spatial Analysis (MSA) \\ in the Program of \\ Spatial Analysis
}

Bachelor of Arts (BA) in Geographic Information Science

Toronto, Ontario, Canada, 2017

(C) Anna Racovali, 2017 


\section{Author's Declaration}

I hereby declare that I am the sole author of this research paper. This is a true copy of the MRP, including any required final revisions.

I authorize Ryerson University to lend this MRP to other institutions or individuals for the purpose of scholarly research.

I further authorize Ryerson University to reproduce this MRP by photocopying or by other means, in total or in part, at the request of other institutions or individuals for the purpose of scholarly research.

I understand that my MRP may be made electronically available to the public. 


\begin{abstract}
This paper explores alternative methods in which an urban walkability score may be determined. Walk Score is a popular urban accessibility index which determines the walkability of a neighbourhood or specific address by measuring the proximity of the location to nearby services and amenities. Traditional walkability scores, such as Walk Score, are limited because of their inability to vary the importance of being in proximity to certain services and amenities. Multi-criteria decision analysis (MCDA) techniques, specifically simple additive weighting (SAW) and ordered weighted averaging (OWA), provide a geographic approach to determining the walkability of an area and allow users to determine the weights of importance of all services and amenities. MCDA-based walkability scores were calculated and compared to one another and to Walk Score. Both SAW and OWA methods created similar walkability indexes for dissemination areas throughout Toronto. However, the MCDA results could not be directly compared to Walk Score, as there was a significant difference between the value ranges of the scores. Thus, the 140 Toronto neighbourhoods were ranked from most to least walkable for the MCDA-based methods and Walk Score, based upon each method's respective scores. Upon comparison, it was evident that both Walk Score's methodology and the MCDA-based methodologies resulted in similar outcomes of walkability rankings for Toronto neighbourhoods.
\end{abstract}




\section{Acknowledgements}

First, I would like to thank Professor Claus Rinner for all of his help and support throughout the entire process of writing this research paper. I am also thankful for the support of my fellow students in the Master of Spatial Analysis program for their useful insights and comments during the analysis process. Lastly, I would like to give a special thanks to my parents for their complete support and patience throughout this past year and many years before. 
Table of Contents

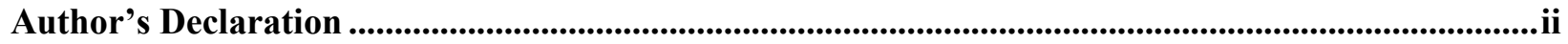

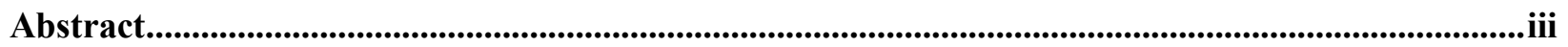

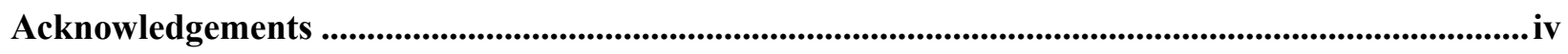

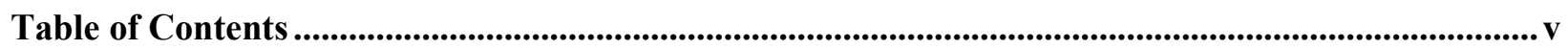

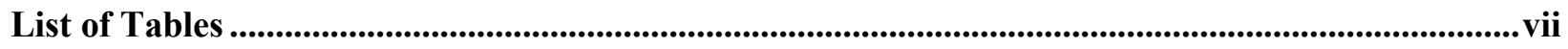

List of Figures..........................................................................................................................................................viii

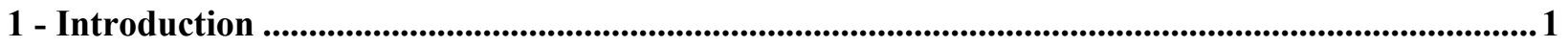

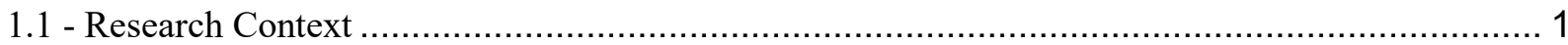

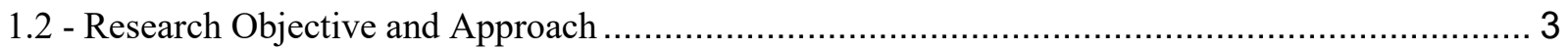

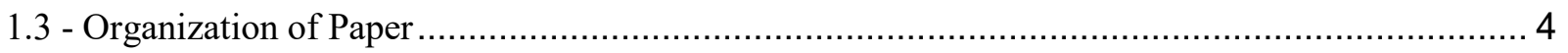

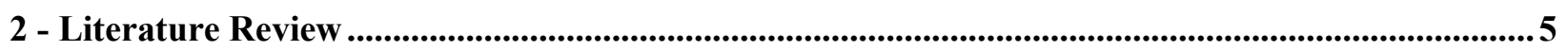

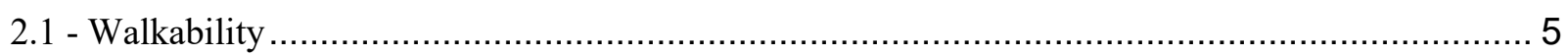

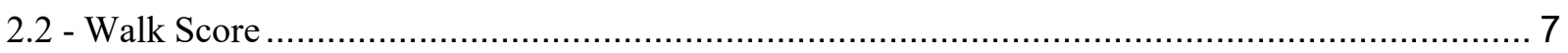

2.3 - Multi-Criteria Decision Analysis Techniques ....................................................... 10

3 - Application Scenario and Data.............................................................................................................14

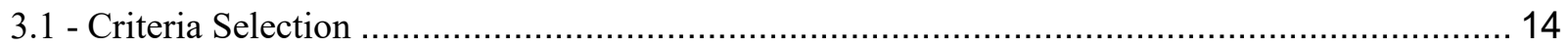

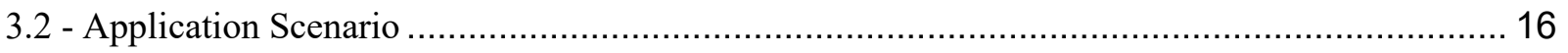

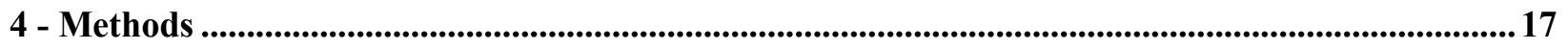

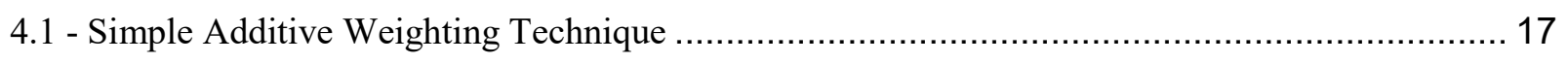

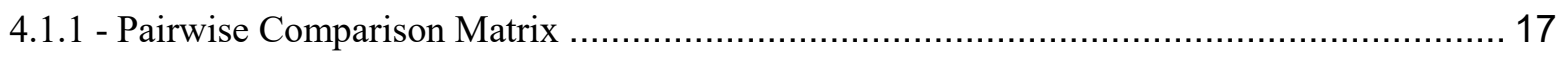

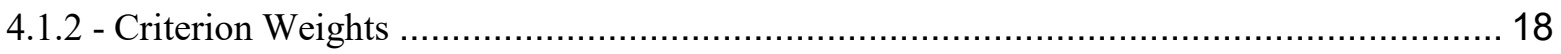

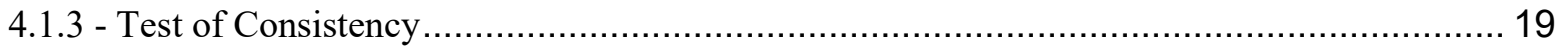

4.1.4 - Determining Simple Additive Weighting Scores .................................................. 20

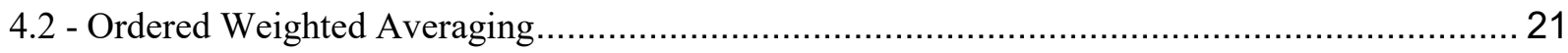

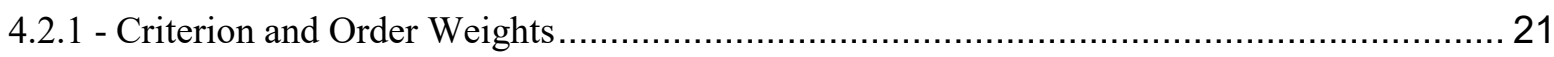

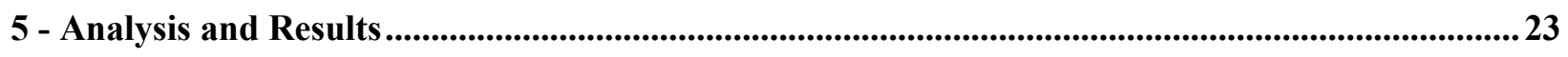

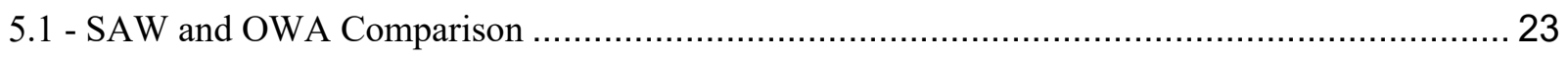

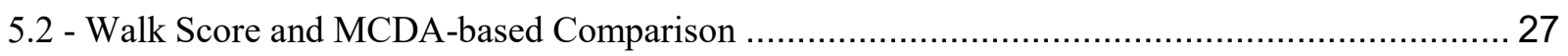

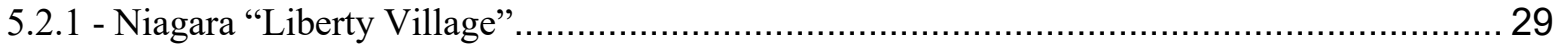

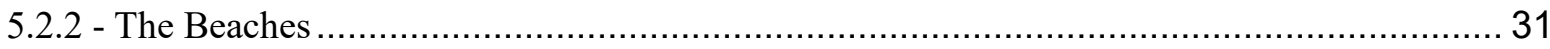

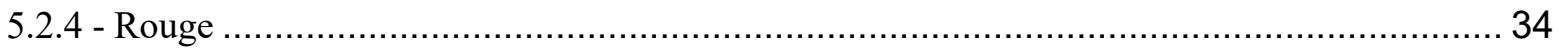

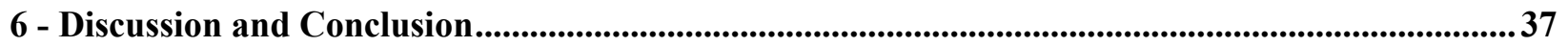




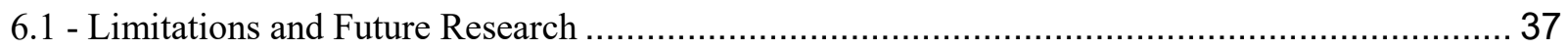

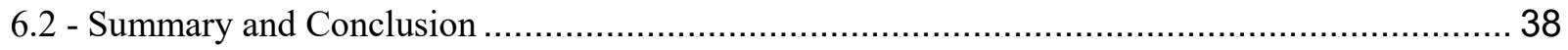




\section{List of Tables}

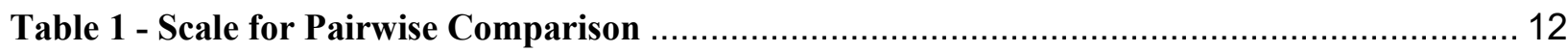

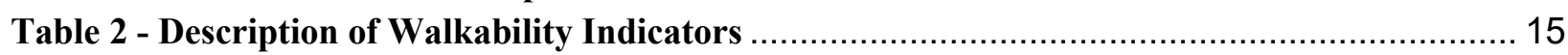

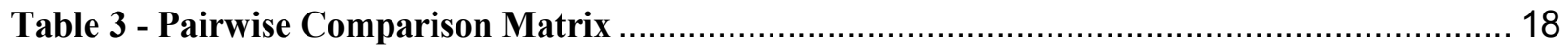

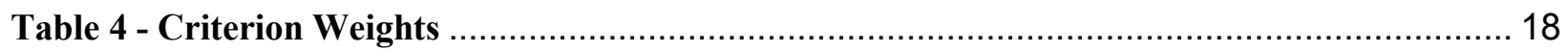

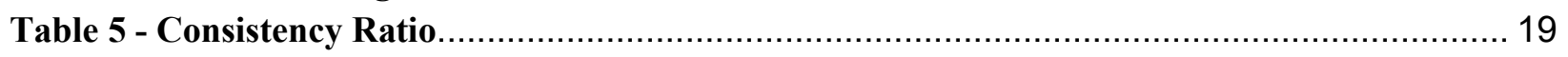

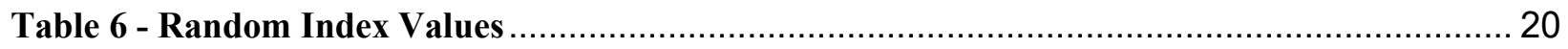

Table 7 - Values of the $\alpha$ parameter for different linguistic quantifiers ................................... 22

Table 8 - Order weights using the linguistic quantifier "many" .......................................... 22

Table 9 - Top 10 Most Walkable DAs in Toronto Based on SAW and OWA Methods................. 25

Table 10 - Bottom 10 Least Walkable DAs in Toronto Based on SAW and OWA Methods ........... 26

Table 11 - Walk Score Neighbourhood Ranking of Walkability ......................................... 28 


\section{List of Figures}

Figure 1 - SAW Walkability Score of Toronto Dissemination Areas .......................................... 24

Figure 2 - OWA Walkability Scores of Toronto Dissemination Areas.......................................... 24

Figure 3 - Bivariate LISA Cluster Map of SAW and OWA Scores ...................................... 26

Figure 4 - Walk Score Heat Map of Toronto's Walkability Scores......................................... 27

Figure 5 - Niagara Neighbourhood SAW Walkability Scores ................................................. 30

Figure 6 - Niagara Neighbourhood OWA Walkability Scores.................................................. 31

Figure 7 - The Beaches Neighbourhood SAW Walkability Scores .......................................... 32

Figure 8 - The Beaches Neighbourhood OWA Walkability Scores........................................ 33

Figure 9 - Rouge Neighbourhood SAW Walkability Scores ............................................. 35

Figure 10 - Rouge Neighbourhood OWA Walkability Scores............................................. 35 


\section{1 - Introduction}

\section{1 - Research Context}

Throughout the past century, advancements in transportation technology, including electric streetcars, automobiles, and highways, have had a serious negative impact on the environments of pedestrians (Forsyth and Southworth, 2008). These upgrades in transportation systems have created barriers for pedestrians, thus encouraging them to use these new methods of transit to reach their destinations. The rapid decline in pedestrian-oriented environments has created neighbourhoods that lack community engagement and public life (Forsyth and Southworth, 2008). This shift created automobile-oriented environments, allowing urban planners to design communities assuming every family would use a car to travel to all services and amenities needed. In the 1950s, after the Second World War, a significant portion of Canada's population began moving into cities. By 1971, approximately $75 \%$ of the country's population lived in metropolitan areas (Bourne and Ley, 1993). This rapid movement of population encouraged urban planners to begin designing new developments of homes on the outer edges of cities. This expansion was known as 'urban sprawl' and created a much lower density of homes than found in downtown areas. Bourne and Ley (1993) defined urban sprawl as “... spatially uncoordinated processes of development, an anarchic cultural landscape made possible by the increasingly widespread ownership and use of automobiles (235)." These neighbourhoods had over-sized roads and limited sidewalks. This lack of connectivity among suburban neighbourhoods, greatly reduced the ability of pedestrians to access all necessary services and amenities (Forsyth and Southworth, 2008).

The creation of suburbs and automobile-oriented environments was deemed successful for decades due to people's willingness to depend on automobiles. However, in recent years, there has been a growing acknowledgement of environmental issues, such as air quality, and health issues, including increasing sedentary lifestyles, stemming from lack of pedestrian opportunities. Public transportation systems have become increasingly more popular and greater interest in pedestrian-focused areas have taken hold. Urban planners have begun promoting more walkable cities with the support of environmental and health studies, through government regulations and incentives (Forsyth and Southworth, 2008). Pedestrian advocates and 
environmentalists began discussing the notion of 'green' modes of transportation, such as walking and cycling. These methods help to reduce road congestion and limit air and noise pollution. Walking also has many individual health benefits, including cardiovascular fitness, reduced stress, and higher mental alertness and creativity. Additionally, the ability to walk to school, work or other services improves social engagement, allowing seniors and children to have equal access to services and amenities (Forsyth and Southworth, 2008).

These benefits represent just a few of the positive outcomes that are possible when living in a walkable area. Urban planners have begun searching for ways that automobile-oriented environments within cities can be shifted to be pedestrian-focused. The first key step in this process is identifying areas within cities that need improving. Urban accessibility scores measure the walkability of a neighbourhood by taking into account the proximity to nearby services and amenities. Walk Score is a popular web-based urban accessibility index that determines the walkability of locations at varying geographic scales in United States, Canada, and Australia (Walk Score, 2017). This type of index allows urban planners, home buyers and government officials to understand which areas within cities are more walkable than others, thus determining where improvements are necessary. Walk Score has been used by many urban planners and officials when making these decisions. However, limited work has been done in exploring the methods, by which Walk Score determines a location's walkability. Two prominent studies that have previously validated Walk Score's methodology used statistical approaches, using Pearson and Spearman correlations and were both done by Lucas J. Carr, Shira I. Dunsiger and Bess H. Marcus (Carr et al., 2010, Carr et al., 2011).

Although Walk Score includes proximity as a key factor, no research was found that validated Walk Score using a geographic approach. Multi-criteria decision analysis (MCDA) techniques are commonly known for their decision-making capabilities. Malczewski (1999) states that "multi-criteria decision-making problems use a set of alternatives that are evaluated based on conflicting and incommensurate criteria" (81). MCDA is dependent on decision makers who consider a set of alternatives, potential outcomes, and aim to determine which of these is the most suitable. Each alternative fulfills the necessary criteria at different scales. The alternative with the highest score is deemed the most suitable, in context of this study, this alternative is deemed the most walkable by including the greatest number of walkability factors. 
MCDA contains numerous sub-methods including Simple Additive Weighting (SAW) and Ordered Weighted Averaging (OWA). SAW, also known as Weighted Linear Combination (WLC), is one of the most commonly used MCDA techniques (Malczewski, 1999). It determines the most optimal alternative by summing the weighted criterion values for each potential outcome. A significant benefit of MCDA methods, when compared to Walk Score's methods, is that decision makers determine the criterion weights, thus determine the importance of each criterion in the decision problem. The alternative with the highest resulting value is determined to be the most suitable. The SAW method has two assumptions: linearity and additivity. The linearity assumption requires that the criterion weights increase by equal units, no matter the level of importance. The additivity assumption implies that there is no correlation amongst the criteria (Malczewski, 1999). Ordered weighted averaging (OWA) has a similar approach to SAW, however it represents fuzzy decision-making situations. OWA uses ordered weights in addition to criterion weights. Thus, the ranking of the criteria plays a role in how they are weighted. Similarly, to the SAW method, the alternative with the highest score is deemed the most suitable (Malczewski, 1999).

\section{2 - Research Objective and Approach}

This research paper aims to understand if there is a significant difference between Walk Score's evaluation of neighbourhoods' walkability and walkability scores created by MCDA techniques. In contrast to Walk Score, MCDA-based methods give users control of what is deemed necessary in their walkable neighbourhood. This is done by allowing users to alter the weights of importance for services and amenities included in the study based on their preferences. This paper explores the use of two widely accepted MCDA techniques, SAW and OWA, to determine the walkability of dissemination areas (DA) and compare them to Walk Score's evaluation.

To perform this comparison, walkability scores were created using both SAW and OWA methods for Toronto DAs. The resulting MCDA-based walkability scores were compared to Walk Score's evaluation for Toronto as a whole and for select neighbourhoods within the city, including Niagara, the Beaches and Rouge. This comparison also aided in recognizing the impacts of using differing methodologies to create walkability scores. 


\section{3 - Organization of Paper}

Chapter 2 provides an overview of the term 'walkability' and the impact of its presence within neighbourhoods. Additionally, it breaks down Walk Score and their methodology for providing an evaluation of walkability. Lastly, an overview of MCDA techniques is provided that explains both SAW and OWA methods. Chapter 3 explains the selected study area and the

process of choosing criteria for the analysis. Chapter 4 provides an overview of the organization of the analysis, the methods used and the ways in which the analysis will aid to identify differences between Walk Score and the MCDA-based evaluations. Chapter 5 highlights the results of the analysis and discusses the relationship between Walk Score and the MCDA-based evaluation. Lastly, Chapter 6 summarizes key findings of the study, discuss the limitations that arose in the study and state further steps that may be suitable in future studies. 


\section{2 - Literature Review}

\section{1 - Walkability}

The ability to walk to one's destination has become an increasingly important quality people look for in their communities. In recent years, people have begun to emphasize the importance of being able to access services by walking as a means of transportation due to its social, environmental, economic and health benefits (Rogers et al., 2010). Socially, the ability to walk to one's destination improves accessibility, the capability of being reached, for a greater population including children and seniors. Additionally, it removes the divide between upper and lower income classes who are now able to access all necessary services without needing a vehicle (Forsyth and Southworth, 2008). Environmentally, walking is considered a "clean" method of transportation as it has minimal environmental impacts. Allowing people, the opportunity to walk to their destination rather than drive removes congestion from roads and reduces noise and air pollution (Forsyth and Southworth, 2008). From an economic standpoint, it is much cheaper for people to walk to services rather than drive as they are minimizing fuel and parking costs. Additionally, there has been a growth in research recognizing businesses favouring pedestrian traffic due to an increase in interaction between businesses and customers (Halstead and Deller, 1997). Lastly, there is a promotion of mental and physical health, encouraging a higher level of physical activity within communities. This notion is important due to the evident growth in sedentary lifestyles that many people have (Forsyth and Southworth, 2008). This is prominent with children and seniors who tend to be less mobile. Thus, high levels of walkability in their local environments is necessary to help encourage physical activity (Gilliland et al., 2009).

Although it is evident that the ability to walk in a community is beneficial, its role in creating safer, more accessible, and socially inclusive neighbourhoods has been a challenge to identify. This is due to the decreasing access pedestrians have had in many cities (Evans, 2009). In recent years, municipal governments have begun to recognize the importance of pedestrianoriented cities. However, there has been minimal work done to create more walkable neighbourhoods. This point is supported by a significant amount of literature that states that many cities continue to lack proper pedestrian accessibility. In a study performed by Hess and Farrow (2014), 250 participants from 8 high-rise neighbourhoods situated throughout Toronto were surveyed and asked about the walkability of their neighbourhoods. $56 \%$ of participants 
noted that they do not have a driver's license while $42 \%$ stated that their household does not own a vehicle (Hess and Farrow, 2014). Many residents in high-rise neighbourhoods are dependent on the ability to walk to services or have access to transportation systems that will take them to their destination.

Many of these people choose to walk to the grocery store or to work, however, they often are faced with hostile pedestrian environments (WHO, 2012). When communities of apartment buildings were first designed, there was a significant assumption that all residents would own vehicles and rely on them to travel to their destinations (Hess and Farrow, 2014). This focus has left pedestrians with unsafe walking environments. Pedestrians face larger travel times due to the lack of accessibility to services and amenities, thus informal connections are created through private properties and fences (Hess and Farrow, 2014). This often occurs because of the dangers of walking along major arterial roads. Many of the roads within the study areas had narrow sidewalks with very few crosswalks and traffic lights (WHO, 2012). These dangers have led those who live in these areas to have varying views of their community's walking conditions. Parents are often fearful of letting their children walk along major roads because of the lack of safety and regulation of traffic. Young people who were surveyed expressed anxiety when walking in car-oriented neighbourhoods due to the lack of lighting and other pedestrians (Hess and Farrow, 2014). 56\% of women and $73 \%$ of people over the age of 65 who were surveyed expressed that they avoid walking at night because of the lack of security measures in place for pedestrians (Hess and Farrow, 2014).

These insecurities and challenges lead people to have a negative perception of their communities. This fact is a significant motivator for cities to improve the walkability and accessibility of their neighbourhoods. Firstly, there are varying types of accessibility, including relative and integral (Bhat et al., 2000). Relative accessibility focuses on the ability to move between two points with ease. While integral accessibility emphasizes the ability to travel from one point to all other points in an area (Bhat et al., 2000). When measuring accessibility of neighbourhoods, this latter definition has been emphasized. Rather than focusing on the accessibility of specific places, urban planners have begun shifting their focus to the overall accessibility each person has within an area (Knox, 1978). Bhat et al. (2000) defines accessibility as "... a measure of the ease of an individual to pursue activities of a desired type, at a desired location, by a desired mode, and at a desired time". This definition emphasizes the focus of 
accessibility on people, rather than the places that they are travelling to. The focus on people's accessibility aids in removing the challenges mentioned by Hess and Farrow (2014). Identifying where informal access points have been made by pedestrians allows urban planners to create formal connections in these areas. Thus, rather than focusing on ensuring there is a route for pedestrians to take to each service and amenity, attention is given to the routes pedestrians want to use and ensuring they are well lit and properly divided from traffic. Making these changes may attract more people to walk rather than drive, thus increasing the number of pedestrians and reducing anxieties expressed by young adults of walking alone in certain areas (Hess and Farrow, 2014).

In addition to the evolving definition of accessibility, the ability to measure it has grown in importance. Quantifying accessibility allows urban planners and municipal governments to measure the quality of transportation systems and the quality-of-life individuals have within certain communities and neighbourhoods (Bhat et al., 2000). Pirie (1979) argues that the ability to measure accessibility allows cities to choose to maintain a certain level of walkability for its citizens. It also makes social and accessibility inequalities more identifiable, allowing officials to understand what improvements are necessary (Pirie, 1979).

\section{2 - Walk Score}

A common use of measured accessibility is developing urban accessibility scores. These scores are calculated to show the overall walkability of an area based on numerous factors. Walk Score is a popular web-based urban accessibility index which provides a score between 0 and 100 , representing a location's walkability to services within proximity to specific address locations. Users are able to determine the walkability of cities, neighbourhoods and even specific addresses throughout the United States, Canada, and Australia (Walk Score, 2017). The Walk Score algorithm measures the distance from address points to 13 amenity categories including grocery stores, coffee shops, restaurants, bars, movie theatres, schools, parks, libraries, book stores, fitness centres, drug stores, hardware stores and clothing stores (Carr et al., 2011). Walk Score typically uses an 'as the crow flies' distance measurement, which measures the straight-line distance between a point and a specific service (Carr et al., 2011). However, the newest version of the program features a network-based algorithm which considers amenities along street networks (Nykiforuk et al., 2017). In addition, Walk Score provides only an estimate of the 
density of services within an area, thus not always depicting an accurate picture of a neighbourhood's walkability. Walk Score is limited in its ability to account for all variables that contribute to a neighbourhood's walkability such as public transportation, street and sidewalk width, block length, intersection density and natural walking barriers such as bodies of water (Carr et al., 2011).

The acknowledgement of Walk Score's limitations has resulted in research on its validity. Limited research has been done, however two significant studies have been performed. First, a validation of the original version of Walk Score, using Rhode Island as the study area (Carr et al., 2010) and secondly a study validating the network-based Walk Score, measuring the walkability of four urban regions in the United States (Duncan et al., 2011). These studies have proven critical as Walk Score continues to play a key role in assessing the walkability of neighbourhoods and the associated levels of physical activity of people within study areas (Nykiforuk et al., 2017).

In the first significant study validating Walk Score's evaluation, Carr et al. (2010) explored the relationship between Walk Score and physical activity environments as a global estimate. Walk Scores were collected for the addresses of 296 participants living in Rhode Island. Additionally, objective measurements of street connectivity, residential density, access to public transit and crime rates were formed using Geographic Information Systems (GIS). These measurements were cross-referenced with people's perception of their environment and Walk Score evaluations (Carr et al., 2010). Lastly, pairwise Pearson correlations were calculated to compare Walk Score to the objective measurements of the study area's walkability. Carr et al. (2010) found that there was a significant correlation between Walk Score and the multiple objective measurements collected for those living in Rhode Island. However, Walk Score also had a positive correlation with reported crimes within the study area, meaning areas of high walkability tended to have higher crime rates. This may be due to the socio-demographics of the study area. Thus, Walk Score does not consider crime rates when determining the walkability of an area. Although, it is important to note that Walk Score had a positive correlation with participant's perception of their physical environment and facilities (Carr et al., 2010). Carr et al. (2010) noted that this perception may in fact be related to the presence of sidewalks and streetlights in high density areas rather than the overall walkability of the study areas. 
Carr et al. (2010) first study focused on the validity of Walk Score in regard to street connectivity, residential density, and crime rates. They continued this research in 2011 by studying Walk Score's ability to estimate access to nearby services and amenities (Carr et al., 2011). Continuing to use Rhode Island as the study area, 379 Walk Score evaluations were collected for both residential and non-residential addresses. 4194 amenities that are located within the study area were accounted for. Geospatial data related to these amenities were collected from open data sources and converted to point shapefiles. Similarly, to Walk Score, a 1-mile buffer was formed around each address location. Walk Score uses a point-based award system based on the distance from an address to amenities within the 1-mile buffer (Carr et al., 2011). Walk Score's point system is separated into five categories: 0-24 = car dependent with no services within walking distance, $25-49=$ car dependent with a few services within walking distance, 50-69 = somewhat walkable with some services and amenities within walking distance, 70-89 = very walkable and able to travel without a vehicle, $90-100=$ walkers' paradise, almost all errands can be performed on foot and many people do not own a car (Carr et al., 2011). Paralleling Walk Score, Carr summed the number of amenities within a 1-mile buffer for each address location, creating his own evaluation of each participant's address walkability. Lastly, Pearson correlations were formed using the sum of the GIS-derived walkability evaluations and the Walk Score evaluations (Carr et al., 2011). The results of the study showed that there was a strong correlation between Walk Score and the individual categories of walkable amenities. Areas of high density appeared to have higher Walk Scores and a higher number of amenities (Carr et al., 2011).

After Walk Score's update to include network-based distances, Duncan et al. (2011) studied Walk Score's validity using the newer version of the urban accessibility score. The objective of the study was to assess the neighbourhood walkability of four US metropolitan areas using geographic indicators with several street network buffer distances. Duncan et al. (2011) aimed to validate the ability of Walk Score to evaluate the walkability of the four study areas with his own study of their walkability. Geospatial data were collected that helped to measure multiple objective indicators of neighbourhood walkability. This data included the number of retail walking destinations (e.g., clothing stores, drug stores and bookstores) per square kilometer, total number of service walking destinations (e.g., post offices, banks, credit unions) per square kilometer, total number of cultural and education walking destinations (e.g., movie 
theatres, schools), median pedestrian route directness (the median of the ratio of distance between two points via road networks and straight line distance between the same two points) and parks per square kilometer. Address data were also collected for each participant in the study areas, along with the Walk Score evaluation of each address. Spearman correlations was calculated, comparing Walk Score and the GIS-based evaluation of walkability (Duncan et al., 2011). The strength of the correlation varied for each neighbourhood walkability indicator at different spatial scales. Walk Score was deemed valid at 400-, 800-, and 1600-metre street network buffers, however the larger the distance band, the higher the correlation. Thus, Walk Score is more suitable to be used when observing walkability at a smaller cartographic scale. (Duncan et al., 2011).

\section{3 - Multi-Criteria Decision Analysis Techniques}

All previous studies of validating Walk Score have used predominantly statistical approaches, such as Pearson and Spearman correlation, to determine the relationship between Walk Score's evaluation and GIS-based evaluations. However, there are numerous other techniques that have yet to be utilized in validating Walk Score. These methods include Multi-Criteria Decision Analysis (MCDA) techniques. MCDA is often used for spatial decision making. Its techniques combine multiple factors to create a composite score of the suitability of numerous locations and thus may be utilized to determine the walkability of neighbourhoods.

MCDA is dependent on a single or group of decision maker(s) who consider a set number of alternatives and aims to determine the 'optimal' alternative considering all factors included in the analysis (Zopounidis, 2002). It is important to note however, that the most suitable location may not be the most optimal in the traditional sense, rather it is a satisfactory option considering the system of values of the decision maker. There are different types of MCDA problems or 'problematics' (Roy, 1996). These include choosing a single alternative, sorting alternatives into groups based on preference order, ranking alternatives from best to worse and describing the performance of each alternative based on their ability to meet criteria (Roy, 1996). The chosen problematic is based upon the context of the MCDA case study and its objective.

The first initial step of the decision-making process is determining the set of alternatives involved in the study. The set may be discrete (a finite number of alternatives) or continuous (an infinite number of alternatives), depending on the context of the study. For both discrete and 
continuous sets of alternatives, the second step of the decision-making process is to compile all relevant factors, known as 'evaluation criteria' (Zopounidis, 2002). These criteria are used to measure the performance of the alternatives and compare them to one another. To compare the performance of the alternatives, the criteria must be aggregated. There are numerous methods in which the criteria can be aggregated thus influencing the results of the decision-making process. Examples of these methods include Simple Additive Weighting methods, Value/Utility Function approaches, Analytic Hierarchy Process, Ideal Point methods and Ordered Weighted Averaging (Malczewski, 1999). Most of these methods require the standardization, or rescaling, of the criterion values to a common numeric range. For this research paper, Simple Additive Weighting (SAW) and Ordered Weighted Averaging (OWA), two of the most common decision rules, will be utilized. To ensure that the decision maker's preferences are accounted for during aggregation, criterion weights and trade-offs can be utilized (Zopounidis, 2002). Each relevant criterion represents a component or aspect of the optimal decision. Their associated weights represent their relative importance (criteria weights) or substitution rate between one criterion and all other criteria (trade-offs) to the decision-process and represent the unique preferences of the decision-maker (Feick and Hall, 2004).

As mentioned earlier, numerous techniques may be used to aggregate criteria to determine optimal locations for MCDA studies. The SAW method, also known as Weighted Linear Combination (WLC), is referred to as the most common technique used for spatial decision making (Afshari et al., 2010). The process begins by the decision maker assigning weights to a set of attributes according to their relative importance. These weights are then multiplied by the scaled values of the alternative related to each attribute. An overall score is produced by summing the products for all attributes. The alternative with the highest score is deemed the most suitable (Malczewski, 1999). There are numerous steps involved when performing a SAW analysis. First, a pair-wise comparison matrix for all relevant criteria is created. Pair-wise comparison matrices are useful due to their ability to compare each criterion to every other criterion individually. There are numerous scales that can be used to compare criteria. Afshari et al. (2010) uses Saaty's 1-9 scale of pair-wise comparison, seen below in Table 1. 


\begin{tabular}{ll}
\hline Intensity of Importence & Definition \\
\hline 1 & Equal importance \\
2 & Equal to moderate importance \\
3 & Moderate importance \\
4 & Moderate to strong importance \\
5 & Strong importance \\
6 & Strong to very strong importance \\
7 & Very strong importance \\
8 & Very to extremely strong importance \\
9 & Extreme importance \\
\hline
\end{tabular}

Soterce: Saaty (1980).

A score based on the scale values is given to each comparison made between the criteria. For each comparison, the decision-maker must decide which one of the two criteria is more important and to what extent (Afshari et al., 2010). The resulting matrix can be used to create a weighted sum matrix, showing the overall importance of each criterion in the decision-making process. The weighted values of each criterion are summed in relation to the alternative they are linked to. The alternatives' summed scores are ranked highest to lowest, highest being the most optimal alternative (Malczewski, 1999).

It is important to note that there are two key assumptions involved in the SAW method. The first assumption is linearity, meaning that the weight of a criterion is increased by equal units, no matter the level of criterion/attribute value (Malczewski, 1999). Secondly, the SAW method assumes additivity, meaning there is no correlation between criteria. If there is a significant correlation between criteria, they must be aggregated into one single criterion (Malczewski, 1999). This may not be possible however, if both criteria are deemed important by decision-makers.

Yager (1988) first introduced OWA aggregation operators. This method uses weighted sums, along with an ordered evaluation of the criteria. The standardized, weighted criterion values must be ordered in descending order for each alternative. A second set of weights, order weights, are then multiplied with the corresponding reordered criterion values (Malczewski and Rinner, 2005). Such that the first-order weight is assigned to the highest criterion value, the second-order weight is assigned to the second highest criterion value, and so on (Drobne and Lisec, 2009). The resulting products are summed for each alternative giving an overall evaluation. The alternative with the highest score is deemed the most suitable. The set of order weights is determined by the number of criteria in the study and the selected linguistic 
quantifiers which are each associated with a specific $\alpha$ parameter. Linguistic quantifiers represent the amount of the weighted criterion values the decision maker wants to take into consideration when determining suitable alternatives (Malczewski and Rinner, 2005). The order weights numerically represent trade-off between different criteria. For example, if all weight is given to the first or last reordered criterion, no tradeoff with other criteria exists. If equal order weights are given, each criterion is considered equally. With equal order weights, the OWA method outcome would be identical to the SAW method outcome. The measure of tradeoff represents the amount of risk the decision-maker(s) is willing to take or avoid. Positive outcomes are weighted higher when decision-maker(s) have a high-risk perception, while those with a lowrisk perception will weight negative outcomes more highly. The differences in risk affect the resulting order weights, thus the resulting suitability of each alternative in the study. 


\section{3 - Application Scenario and Data}

To compare the walkability evaluations of Walk Score and the MCDA techniques, the city of Toronto was used as the study area. Toronto has a variety of urban landscapes, including sprawling suburban neighbourhoods and a densely populated downtown core. Thus, varying levels of walkability will be accounted for in the analysis. For this study, MCDA-based walkability scores were calculated for each dissemination area (DA) within Toronto. DAs were used as they are the smallest geographic units with data made available by Statistics Canada.

\section{1 - Criteria Selection}

There are numerous factors that play a role in determining the walkability of an area. However, in order to objectively compare the Walk Score metric and the MCDA methods, the analysis will include only the factors used by Walk Score provided in their methodology. These factors include proximity to various types of amenities, intersection density and population density. Although literature has stated that crime rates play a role in measuring walkability, they are not included as a factor in this study. Walk Score created a separate score related to crime rates, thus is not included in their walkability evaluation. In order to objectively compare Walk Score and the MCDA-based evaluations, crime rates are excluded from the study.

Data for these criteria were collected from the City of Toronto Open Data Portal. These data included 'Intersection File - City of Toronto', a point shapefile identifying all intersections within Toronto, 'Address Points (Municipal) - Toronto One Address Repository', a point shapefile related to the locations of services and amenities in Toronto and Dinesafe, a table which lists every food establishment in the city. Population data was collected from Statistics Canada's 2016 Census program, providing the population of each DA in Toronto Data was divided into seven categories that represent each walkability indicator used by Walk Score, thus in this analysis. Shapefiles were divided, using the clip function in ArcGIS, by each amenity and service, as listed in Table 2 below. The resulting shapefiles were then aggregated by indicator categories. Resulting in seven shapefiles. The data included within each indicator is provided in Table 2 below. 
Table 2 - Description of Walkability Indicators

\begin{tabular}{|c|c|c|}
\hline Indicator & Description & \\
\hline $\begin{array}{l}\text { Cultural/Recreation } \\
\text { Points of Interest }\end{array}$ & $\begin{array}{ll}\text { o } & \text { Public Art } \\
\text { o } & \text { Murals } \\
\text { o } & \text { Historical Buildings } \\
\text { o } & \text { Cultural Spaces/Parks } \\
\text { o } & \text { Municipal/Regional/ } \\
\text { Provincial Park }\end{array}$ & $\begin{array}{ll}\text { o } & \text { Community Hall } \\
\text { o } & \text { Fairgrounds } \\
\text { o } & \text { Libraries } \\
\text { o } & \text { Museum/Art Gallery } \\
\text { o } & \text { Sport Complex } \\
\text { o } & \text { Concert Hall/Theatre/Cinema } \\
\text { o } & \text { Golf Course }\end{array}$ \\
\hline Food & $\begin{array}{ll}\text { o } & \text { Grocery Stores } \\
\text { o } & \text { Restaurants }\end{array}$ & o Specialty Food Stores \\
\hline Retail & $\begin{array}{l}\text { o Community, Neighbourhood } \\
\text { and Regional Shopping Centres }\end{array}$ & o Commercial Locations \\
\hline $\begin{array}{l}\text { Medical/Professional } \\
\text { Services }\end{array}$ & $\begin{array}{ll}\text { o } & \text { Ambulance/Fire Stations } \\
\text { o } & \text { Hospitals (Private/Public) }\end{array}$ & $\begin{array}{ll}\text { o } & \text { Day Care } \\
\text { o } & \text { Medical/Dental } \\
\text { o } & \text { Federal/Provincial Legislative Offices }\end{array}$ \\
\hline Education & $\begin{array}{l}\text { o Elementary and Secondary Sc } \\
\text { Toronto Catholic District School }\end{array}$ & $\begin{array}{l}\text { Dols (Toronto District School Board and } \\
\text { oard) }\end{array}$ \\
\hline Transportation & $\begin{array}{ll}\text { o } & \text { Transit Stops } \\
\text { o } & \text { Sidewalks }\end{array}$ & o Bike Lakes \\
\hline Intersection & Every intersection in the city & Toronto \\
\hline Residential & Population of each dissemina & $n$ area in the city of Toronto \\
\hline
\end{tabular}

To objectively compare the amount of services and amenities to the amount of intersections within a DA, the density of each indicator for each DA was calculated. In order to perform this step, a shapefile of Toronto's DA boundaries was retrieved from Toronto's Open Data website. The density was determined by summing the number of locations of an indicator within each DA by the area of each DA. By measuring criteria by their density in a DA, the resulting evaluations show the potential capacity of services in the area. The higher the density of services and amenities, the greater number of locations within a DA, thus suggesting that the population in that area has a greater chance of receiving service from these locations within close proximity. 


\section{2 - Application Scenario}

Each of the criterion used for the analysis play a key role in the development of walkable neighbourhoods.

- Medical/Professional Services: A key indicator for the safety and well-being of people. These services include emergency services, medical and dental offices, as well as day care centres.

- Education: A key factor for people with children. Parents often would like their children to be able to walk to school, thus reducing congestion on roads from parents driving and school buses. This indicator includes both elementary and secondary schools.

- Cultural and Recreational Points of Interest: This indicator encompasses a wide range of amenities including movie theatres, libraries, museums, public art, concert halls, sport complexes and many more. These amenities play a key role in attracting residents from nearby and far to the area.

- Retail: This indicator includes clothing, books and pharmacy/drug stores and shopping centres. It does not include food stores as it is its own indicator, based on Walk Score's terminology. The presence of retail businesses allows people to purchase goods they need without requiring a vehicle or transportation.

- Food: A critical indicator, people visit grocery stores, bake shops, coffee shops, etc. daily. The greater number of food stores in an area suggests that people will have to travel a shorter distance from food stores to home with groceries.

- Transportation: This indicator is vital for allowing people to access other areas of the city. A higher density of transit stops, bike lanes and sidewalks give more people accessibility to all necessary points of interest.

- Intersection Density: This indicator represents the connectedness of a neighbourhood. A higher intersection density suggests that pedestrians have a greater number of accessible routes to choose from to get to their destinations.

- Residential Density: A higher population density often attracts a larger number of services and businesses to an area due to the potential in increased revenue. 


\section{4 - Methods}

\section{1 - Simple Additive Weighting Technique}

The first MCDA technique used is the Simple Additive Weighting (SAW) method. This technique is based on criterion weights that reflect the importance of each criterion within the set of alternatives. In this study, alternatives are defined by the 3,702 dissemination areas found within the city of Toronto. An evaluation score is determined for each alternative by multiplying the standardized values of each criterion by its associated weight. These scores are summed for all criteria. The alternative with the highest score is deemed the most suitable, for this study it is deemed the most walkable.

\subsection{1 - Pairwise Comparison Matrix}

The SAW method can be used with a pairwise comparison matrix to determine the criterion weights, which represent the level of importance of each criterion involved in the analysis. First developed by Saaty (1980), the pairwise comparison method was initially associated with the Analytic Hierarchy Process (AHP), another MCDA technique. Saaty developed a scale with values from 1 to 9 , used to compare the importance of criteria one by one. This scale can be seen in Table 1 above.

When two criteria are compared, the decision maker must decide how much more important criterion A is than criterion B. This relationship is represented by a level of importance as mentioned. Additionally, when completing the pairwise comparison matrix, as seen below in Table 3, each comparison must be performed inversely as well. The pairwise comparison method assumes that when comparing criterion B to criterion A, its importance ranking is the inverse of the importance ranking of criterion A to criterion B (Saaty, 1980). For this research, decisions on the level of importance for each criterion comparison were determined from the point of view of a resident who is looking for ideal conditions for all necessary travelling conditions, without needing a vehicle. It is important to note that these weights are subjective, thus may have different results from a different decision maker's point of view. 
Table 3 - Pairwise Comparison Matrix

\begin{tabular}{l|r|r|r|r|r|r|r|r}
\hline & \multicolumn{1}{|c|}{ Services } & Education & Cultural/POI & Retail & Food & Intersection & Residential & Transportation \\
\hline Services & $\mathbf{1 . 0 0}$ & 4.00 & 2.00 & 4.00 & 2.00 & 2.00 & 2.00 & 1.00 \\
\hline Education & 0.25 & $\mathbf{1 . 0 0}$ & 0.50 & 0.50 & 1.00 & 0.33 & 2.00 & 0.17 \\
\hline Cultural/POI & 0.50 & 2.00 & $\mathbf{1 . 0 0}$ & 2.00 & 0.50 & 1.00 & 2.00 & 0.13 \\
\hline Retail & 0.25 & 2.00 & 0.50 & $\mathbf{1 . 0 0}$ & 1.00 & 3.00 & 3.00 & 0.25 \\
\hline Food & 0.50 & 1.00 & 2.00 & 1.00 & $\mathbf{1 . 0 0}$ & 4.00 & 3.00 & 0.33 \\
Intersections & 0.50 & 3.00 & 1.00 & 0.33 & 0.25 & $\mathbf{1 . 0 0}$ & 1.00 & 0.33 \\
\hline Residential & 0.50 & 0.50 & 0.50 & 0.33 & 0.33 & 1.00 & $\mathbf{1 . 0 0}$ & 0.25 \\
\hline Transportation & 1.00 & 6.00 & 8.00 & 4.00 & 3.00 & 3.00 & 4.00 & $\mathbf{1 . 0 0}$ \\
\hline Sum & 4.50 & $\mathbf{1 9 . 5 0}$ & $\mathbf{1 5 . 5 0}$ & $\mathbf{1 3 . 1 7}$ & $\mathbf{9 . 0 8}$ & $\mathbf{1 5 . 3 3}$ & $\mathbf{1 8 . 0 0}$ & $\mathbf{3 . 4 6}$ \\
\hline
\end{tabular}

\subsection{2 - Criterion Weights}

Each element of the comparison matrix is divided by their respective column totals, creating a normalized matrix, as seen in Table 4 below. Each criterion weight is calculated by finding the average of each row in the matrix, i.e. by summing the normalized matrix elements in each row and dividing by 8 , the number of criteria in the study (Malczewski, 1999). The resulting weights are shown in the last column of Table 4. Based on the pairwise comparison matrix, Transportation is deemed the most important criterion with a weight of 0.30 . Additionally, Residential (density) and Education are the lowest weighted criteria with weights of 0.05 and 0.06 respectively.

\section{Table 4 - Criterion Weights}

\begin{tabular}{|c|c|c|c|c|c|c|c|c|c|}
\hline & Services & Education & Cultural/POI & Retail & Food & Intersection & n Residential & Transportation & Weights \\
\hline Services & 0.22 & 0.21 & 0.13 & 0.30 & 0.22 & $2 \quad 0.13$ & \begin{tabular}{|l}
$3 \quad 0.11$ \\
\end{tabular} & 0.29 & 0.20 \\
\hline Education & 0.06 & 0.05 & 0.03 & 0.04 & 0.11 & 0.02 & 0.11 & 0.05 & 0.06 \\
\hline Cultural/POI & 0.11 & 0.10 & 0.06 & 0.15 & 0.06 & 0.07 & 0.11 & 0.04 & 0.09 \\
\hline Retail & 0.06 & 0.10 & 0.03 & 0.08 & 0.11 & 0.20 & 0.17 & 0.07 & 0.10 \\
\hline Food & 0.11 & 0.05 & 0.13 & 0.08 & 0.11 & 0.26 & 0.17 & 0.10 & 0.13 \\
\hline Intersection & 0.11 & 0.15 & 0.06 & 0.03 & 0.03 & 0.07 & 0.06 & 0.10 & 0.07 \\
\hline Residential & 0.11 & 0.03 & 0.03 & 0.03 & 0.04 & 0.07 & 0.06 & 0.07 & 0.05 \\
\hline Transportation & 0.22 & 0.31 & 0.52 & 0.30 & 0.33 & 0.20 & 0.22 & 0.29 & 0.30 \\
\hline Sum & 1.00 & 1.00 & 1.00 & 1.00 & 1.00 & 1.00 & 1.00 & 1.00 & 1.00 \\
\hline
\end{tabular}




\subsection{3 - Test of Consistency}

It is critical to determine if the comparisons the decision maker has created in previous steps are consistent. This consistency can be determined by multiplying each criterion weight by its associated column of pairwise comparison matrix elements. For example, the (medical/professional) services weight of 0.19 is multiplied by all values in the (medical/professional) services column in the original pair-wise comparison matrix. The resulting values can be seen below in Table 5. The values are summed for each row then divided by each criterion row's associated weight (Malczewski, 1999). The resulting values represent the consistency vector values, these values are essential to measure the extent, to which the decisionmaker has been consistent in their defined weights.

Table 5 - Consistency Ratio

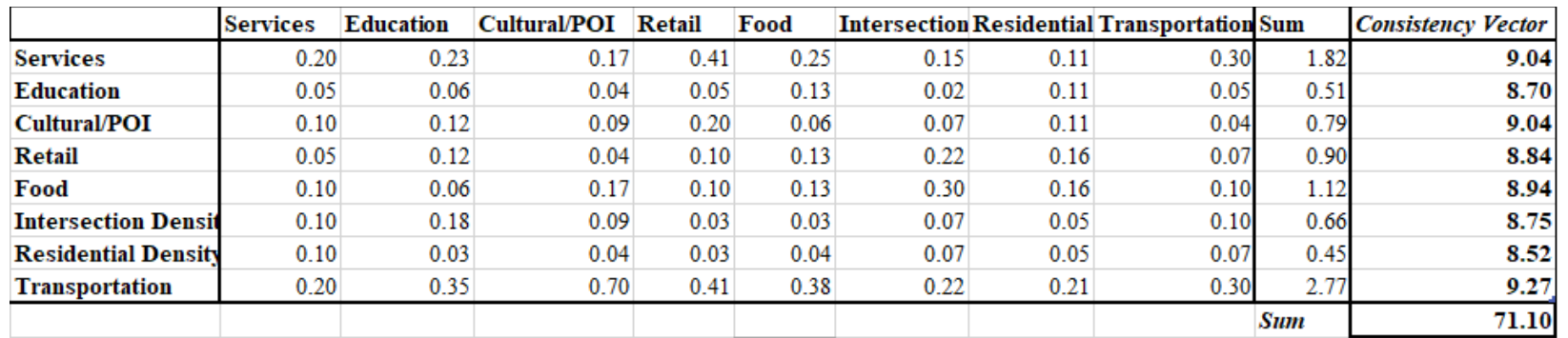

The next step includes determining the value of lambda $(\chi)$. It is calculated by simply averaging the consistency vector values. The resulting lambda value for this study is 8.95 . The consistency index (CI) determines the level of consistency the comparisons have. If $\chi \geq n, \mathrm{n}$ representing the number of criteria in the study, the consistency index assumes the comparison matrix is positive. The comparison matrix is deemed consistent if $\chi=n$. Thus, the difference between $\chi$ and $n$ represents the degree of inconsistency in the matrix (Malczewski, 1999). Formally, the consistency index is calculated for this study as follows:

$$
C I=\frac{\lambda-n}{n-1}=\frac{8.95-8}{8-1}=0.13
$$

The final step of testing the comparison values is to determine the consistency ratio (CR), using the consistency index and random index. The random index (RI) is dependent on the number criteria involved in the study and represents the consistency index of randomly generated pairwise comparison matrices (Malczewski, 1999). For this study, RI $=1.41$, as shown in Table 6 below. 


\section{Table 6 - Random Index Values}

\begin{tabular}{|c|c|}
\hline Size of matrix & Random consistency \\
\hline 1 & 0 \\
\hline 2 & 0 \\
\hline 3 & 0.58 \\
\hline 4 & 0.9 \\
\hline 5 & 1.12 \\
\hline 6 & 1.24 \\
\hline 7 & 1.32 \\
\hline 8 & 1.41 \\
\hline 9 & 1.45 \\
\hline 10 & 1.49 \\
\hline
\end{tabular}

The consistency ratio is the final testing of the comparisons consistency. If $\mathrm{CR}<0.10$, the ratio indicates that the pairwise comparisons have a reasonable level of consistency and can be used in the following steps of the SAW method. If $\mathrm{CR} \geq 0.10$, the pairwise comparison is deemed inconsistent, thus the comparison values must be reassessed (Malczewski, 1999). The consistency ratio is determined by dividing the consistency index by the random index, as seen in the following equation:

$$
C R=C I / R I=0.13 / 1.41=0.09
$$

The resulting consistency ratio for this study is 0.09 . This value is less than 0.10 , thus deeming our pairwise comparison values consistent.

\subsection{4 - Determining Simple Additive Weighting Scores}

Once the criterion weights have been determined and tested for consistency, they must be applied to each alternative. Each criterion is standardized using the score range procedure (Malczewski, 1999). This step is performed within ArcGIS software. For this study, decision makers want to maximize the presence of each criterion, benefiting from a higher presence of each criterion. Thus, the benefit criteria equation is used to standardize each criterion. The equation is as follows:

$$
x_{i j}^{\prime}=\frac{x i j-x_{j}^{\min }}{x_{j}^{\max }-x_{j}^{\min }}
$$

where $X^{\prime}{ }_{i j}$ is the standardized score for the $i$ th alternative and the $j$ th criterion. $X_{j}^{\max }-$ $X_{j}^{m i n}$ represents the range of each criterion's values and $X_{i j}$ is the raw score. The values of the 
standardized score ranges from 0 to 1 . The higher the criterion values, the more attractive they are in the study (Malczewski, 1999).

Once each criterion is standardized, they are each multiplied by their respective weight, previously calculated using the pairwise comparison matrix. The overall score for each alternative is calculated by summing the weighted standardized layers. The alternative with the highest resulting score is deemed the most walkable.

\section{2 - Ordered Weighted Averaging}

The second MCDA method used in this study is the Ordered Weighted Averaging (OWA) technique. Similarly to the SAW method, criterion weights play a key role in determining the most suitable alternatives. In addition, order weights are used. They are associated with the criterion values on a location-by-location basis (Rinner and Malczewski, 2002). Order weights are assigned to individual location's standardized weighted criterion values in decreasing order. This association does not consider which criterion the values come from. This ordering step is essential to the OWA method and separates it from the SAW method.

\subsection{1 - Criterion and Order Weights}

Both criterion weights and order weights are necessary to perform the OWA method. The resulting scores of this method are compared to the alternative scores of the SAW method, thus the same criterion weights are used in the OWA method. The difference between the two methods is the application of order weights.

Order weights are associated with linguistic quantifiers, which represent the translation of language specifications to numerical values (Kickert 1978; Munda 1995). Order weights can be determined using either absolute or relative quantifiers. For this study, relative quantifiers are used. This class of quantifiers are related to imprecise proportions, using terms such as a few, half, many, etc. (Rinner and Malczewski, 2005). Yager (1996) created the following method for defining unit intervals based on specified parameters:

$$
\text { Quantifiers }(p)=p^{\alpha}, \alpha>0 .
$$

where $\alpha$ is the degree of inclusion. The value of $\alpha$ is determined by the decision-maker, thus determining the amount of the criteria they would like to be included in the decision. For this study $\alpha=2$, meaning as the decision-maker, we want many of the criteria to be included in the 
process of determining the most walkable areas within the study area. Table 7 below shows the varying $\alpha$ values that can be used in the process.

Table 7 - Values of the $\alpha$ parameter for different linguistic quantifiers

\begin{tabular}{|l|cccccccccc}
\hline Linguistic Quantifiers & At least one & Few & Some & Half & Many & Most & All \\
\hline$\alpha$ & 0.001 & 0.1 & 0.5 & 1 & 2 & 10 & 1000 \\
\hline
\end{tabular}

The $\alpha$ parameter is used to determine order weights for the study. Order weights are dependent on the number of criteria in the study and the degree of inclusion wanted of all criteria (Rinner and Malczewski, 2005). Thus, the following equation is used to determine the order weights:

$$
v_{j}=\left(\frac{j}{n}\right)^{\alpha}-\left(\frac{j-1}{n}\right)^{\alpha}, \text { for } j=1,2 \ldots n .
$$

Using this equation, order weights for each ranking position are calculated. For this study, 8 order weights are determined, as stated in Table 8 below.

Table 8 - Order weights using the linguistic quantifier "many"

\begin{tabular}{|c|c|}
\hline Order Position & Weights \\
\hline $\boldsymbol{v}_{1}$ & 0.015625 \\
\hline $\boldsymbol{v}_{2}$ & 0.046875 \\
\hline $\boldsymbol{v}_{3}$ & 0.078125 \\
$\boldsymbol{v}_{4}$ & 0.109375 \\
\hline $\boldsymbol{v}_{5}$ & 0.140625 \\
$\boldsymbol{v}_{6}$ & 0.171875 \\
\hline $\boldsymbol{v}_{7}$ & 0.203125 \\
\hline $\boldsymbol{v}_{8}$ & 0.234375 \\
\hline SUM & 1.000000 \\
\hline
\end{tabular}

Once the order weights are determined, the standardized weighted criterion values for each alternative, in this case each dissemination area, are sorted in descending order. Within each alternative, the standardized weighted criterion with the highest value is multiplied by the first order weight, $v_{1}$. This process is repeated by multiplying the second highest value by the second order weight, $v_{2}$, and so on (Malczewski, 1999). Once each standardized weighted criterion is multiplied by its associated order weight and summed for each alternative, the alternative with the highest resulting score is deemed the most walkable. 


\section{5 - Analysis and Results}

Upon completion of the analysis, walkability scores of both SAW and OWA methods are compared. Additionally, the MCDA-based walkability evaluations are compared to the evaluations created by Walk Score. This comparison is performed for Toronto as a whole and three specific neighbourhoods within the city, including Niagara, the Beaches and Rouge.

\section{1 - SAW and OWA Comparison}

The completion of the SAW and OWA methods created walkability scores for all 3,702 DAs within the city of Toronto. The resulting walkability scores are quite small in value. This outcome is not a surprise as the size of each DA is relatively small, limiting the number of services and amenities that may be located within each DA. Statistics Canada describes DAs as having a population size between 400-700 people. Additionally, their boundaries follow urban features such as roads, railways, water features and power transmission lines. DAs are limited in size to a maximum of 99 city blocks (Statistics Canada, 2017). It is important to note that due to the resulting evaluation's lack of units of measurement, the raw scores cannot be compared directly. Only the resulting walkability rankings can be compared to one another.

Visual observations of the resulting SAW and OWA walkability scores by DA show limited differences. Maps of the spatial distribution of the scores are shown in Figure 1 and 2 below for SAW and OWA evaluations respectively. These maps were created using a quantile classification method, thus each class contains the same number of DAs. When comparing maps, if a DA appears to have changed classes from one method to the other, that DA will have changed to a higher or lower walkability score (relative to other DAs within the same evaluation). DAs located in downtown Toronto tend to have higher walkability scores clustered together and the further the DA is from downtown, the lower its associated score. Although, it is important to note that DAs along major commercial streets throughout the city tend to have higher walkability scores. This is due to the increased number of services and amenities along these roads. It appears within each of Toronto's 140 neighbourhoods, there is at least one DA with a higher walkability score. This is shown to be true for both MCDA-based methods. 


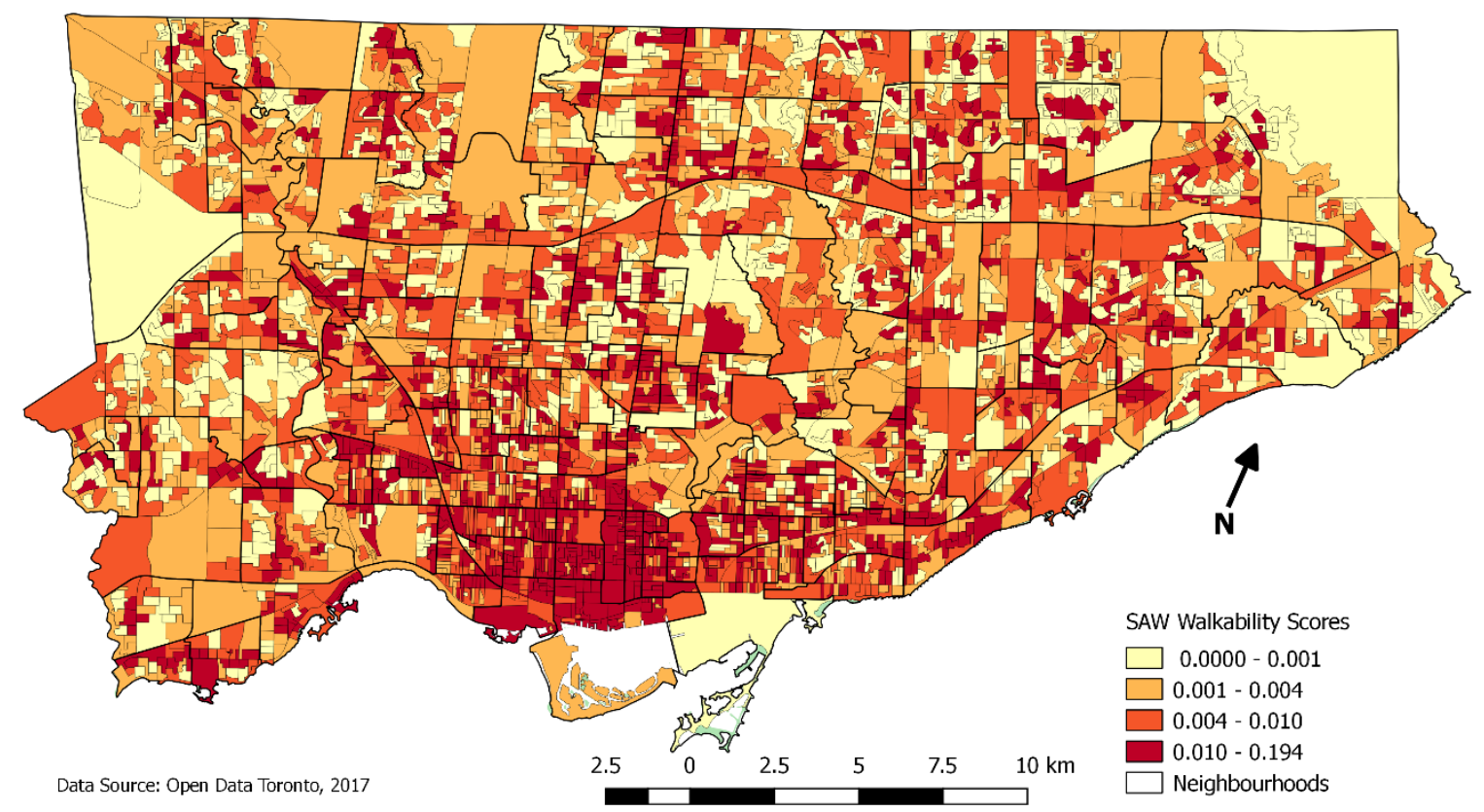

Figure 1 - SAW Walkability Score of Toronto Dissemination Areas

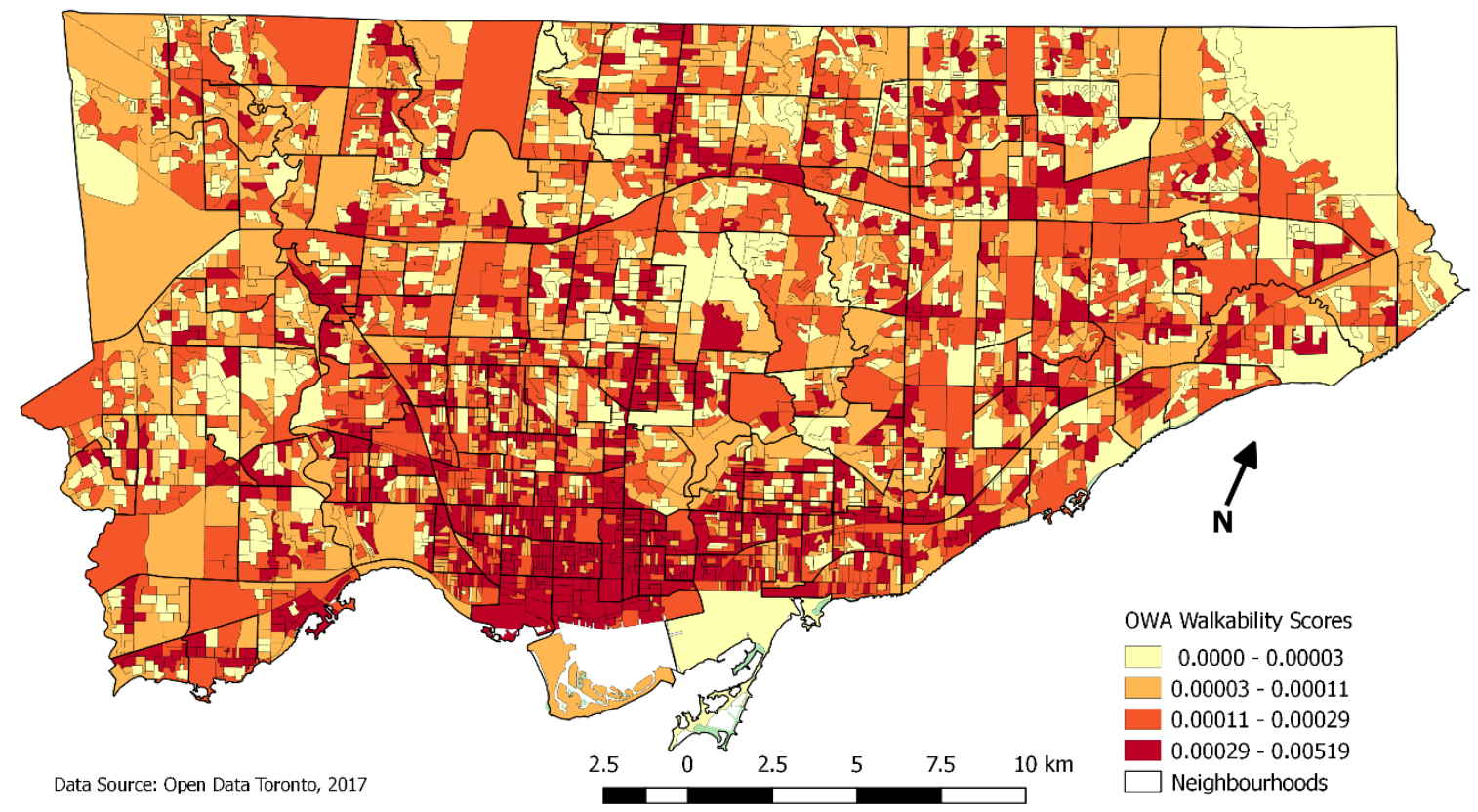

Figure 2 - OWA Walkability Scores of Toronto Dissemination Areas

When comparing the rankings of DAs between both MCDA-based methods, there are noticeable differences. This can be seen in Table 9 below, which shows the top 10 most walkable DAs, 
based on the MCDA-based methods, and the neighbourhoods in which each DA is located. Many DAs are within both SAW and OWA top 10 rankings. The rankings include DAs from various neighbourhoods. However, many of the neighbourhoods listed are centrally located, or to the North and West. The Church-Yonge Corridor is one of the more walkable neighbourhoods, with three of its DAs included in both top 10 rankings.

Table 9 - Top 10 Most Walkable DAs in Toronto Based on SAW and OWA Methods

\begin{tabular}{c|cl|c|c|cl|c}
\hline \multicolumn{3}{c|}{ SAW } & \multicolumn{3}{c}{ OWA } \\
\hline DA & Score & \multicolumn{1}{c|}{ Neighbourhood } & Population & DA & Score & Neighbourhood & Population \\
\hline 35201161 & 0.194 & Weston-Pellam Park & 12,079 & 35201448 & 0.00519 & Trinity-Bellwoods & 16,827 \\
35204620 & 0.172 & Church-Yonge Corridor & 28,427 & 35204620 & 0.00511 & Church-Yonge Corridor & 28,427 \\
35202241 & 0.144 & Oakwood Village & 20,995 & 35204615 & 0.00463 & Church-Yonge Corridor & 28,427 \\
35201448 & 0.142 & Trinity-Bellwoods & 16,827 & 35204100 & 0.00389 & Islington-City Centre West & 38,105 \\
35200855 & 0.140 & Church-Yonge Corridor & 28,427 & 35202818 & 0.00377 & Annex & 29,225 \\
35200901 & 0.117 & Kensington-Chinatown & 18,458 & 35201437 & 0.00352 & Kensington-Chinatown & 18,458 \\
35204624 & 0.113 & Church-Yonge Corridor & 28,427 & 35204755 & 0.00345 & Willowdale East & 45,071 \\
35204311 & 0.106 & Willowdale East & 45,071 & 35204624 & 0.00337 & Church-Yonge Corridor & 24,889 \\
35204688 & 0.099 & Mimico & 26,270 & 35202241 & 0.00336 & Oakwood Village & 20,995 \\
35204755 & 0.095 & Willowdale East & 11,333 & 35201161 & 0.00318 & Weston-Pellam Park & 12,079 \\
\hline
\end{tabular}

Similar comparisons were made when observing the ten least walkable DAs in Toronto, as seen in Table 10 below, ranked from least walkable at the top of the table. Neighbourhoods Morningside, Bayview Village and Rouge are present within both SAW and OWA rankings as having the least walkable DAs. Many of the DAs included in the ten least walkable DAs ranking are present in both MCDA-based rankings, in varying order. Morningside and Bayview Village neighbourhoods each have two DAs that are identified in the least walkable rankings for both SAW and OWA methods. Overall, the neighbourhoods included in this ranking are in northern and eastern parts of Toronto. As opposed to the neighbourhoods identified in the most walkable ranking, which as mentioned, are in downtown Toronto and in northern and western parts of the city. In conclusion, both SAW and OWA methods identified similar DAs as the most and least walkable areas of the city when observing on a visual scale. However, there are noticeable differences when comparing the top and bottom 10 ranked DAs for both SAW and OWA methods. 
Table 10 - Bottom 10 Least Walkable DAs in Toronto Based on SAW and OWA Methods

\begin{tabular}{|c|c|c|c|c|c|c|c|}
\hline \multicolumn{4}{|c|}{ SAW } & \multicolumn{4}{|l|}{ OWA } \\
\hline DA & Score & Neighbourhood & Population & DA & Score & Neighbourhood & Population \\
\hline 35204784 & 0.000089 & Morningside & 17,573 & 35204784 & 0.000001 & Morningside & 17,573 \\
\hline 35200330 & 0.000091 & Bayview Village & 17,678 & 35200330 & 0.000003 & Bayview Village & 17,678 \\
\hline 35204723 & 0.000109 & Rouge & 46,063 & 35204783 & 0.000003 & Morningside & 17,573 \\
\hline 35204783 & 0.000114 & Morningside & 17,573 & 35204723 & 0.000003 & Rouge & 46,063 \\
\hline 35202399 & 0.000149 & Lansing-Westgate & 14,940 & 35204864 & 0.000005 & Henry Fa & 11,333 \\
\hline 35200329 & 0.000150 & Bayview Village & 17,678 & 35204854 & 0.000005 & Parkwoods-Dc & 34,636 \\
\hline 35202097 & 0.000151 & York University Heights & 27,676 & 35202097 & 0.000005 & York University Heights & 27,676 \\
\hline 35200177 & 0.000169 & Steeles & 24,889 & 35203717 & 0.000006 & Rouge & 46,063 \\
\hline 35204342 & 0.000170 & Scarborough Village & 16,597 & 35202399 & 0.000006 & Lansing-Westgate & 14,940 \\
\hline 35204864 & 0.000187 & Henry Farm & 11,333 & 35204342 & 0.000006 & Scarborough Village & 16,597 \\
\hline
\end{tabular}

Although these comparisons are made with only 20 of Toronto's 3,702 DAs, the observations recognized when comparing rankings can be further supported through non-spatial and spatial correlation analysis. The (non-spatial) correlation coefficient between SAW and OWA is 0.982 and indicates a very high degree of correlation. Local Indicators of Spatial Association (LISA) are shown in Figure 3 below. The bi-variate LISA cluster map shows the relationship between SAW and OWA scores and their ranking. As shown in Figure 3, it is evident that both methods identified areas in the downtown core as having high walkability. These areas are labeled as 'high-high', meaning both SAW and OWA evaluations recognized this area as having high

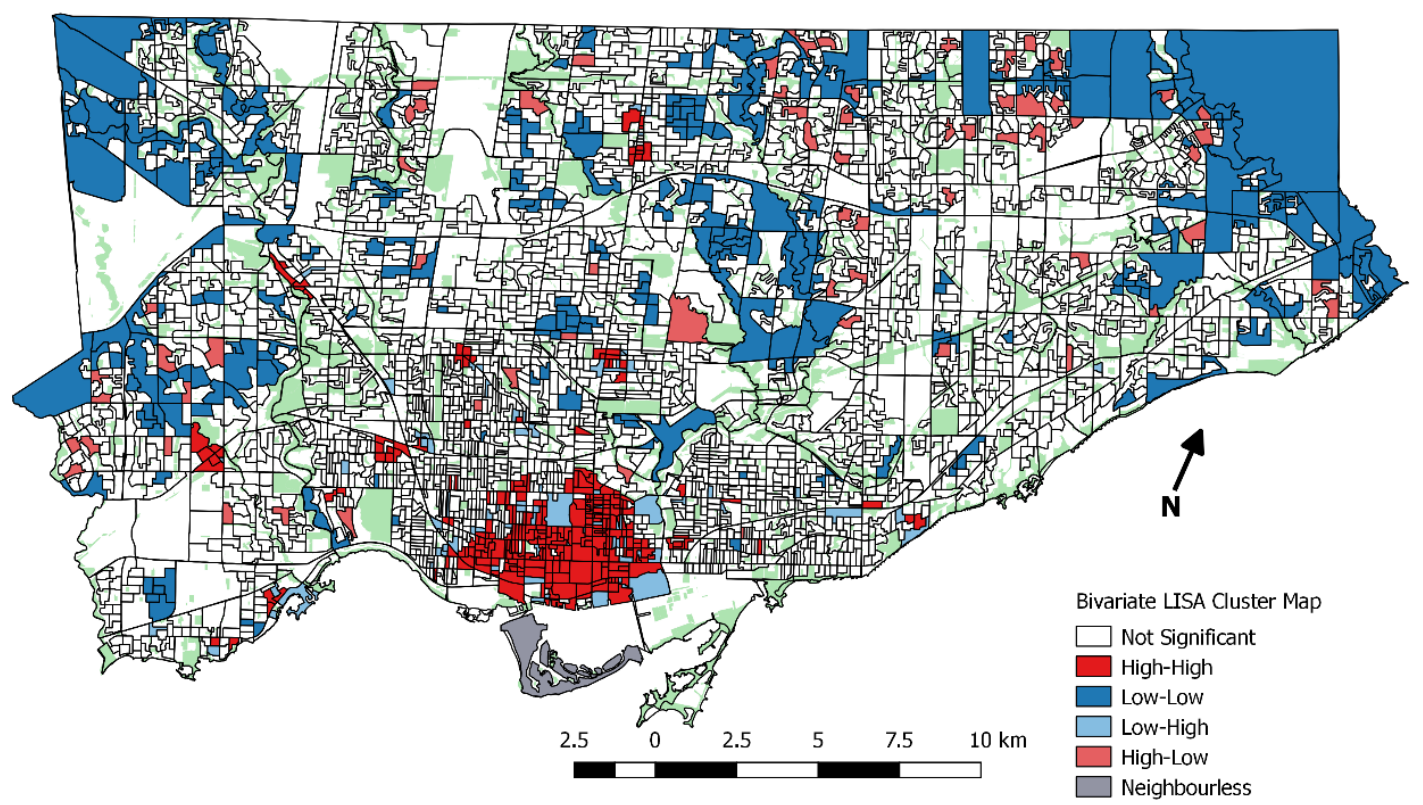

Figure 3 - Bivariate LISA Cluster Map of SAW and OWA Scores 
scores. Additionally, both methods identified DAs located on the outskirts of the City of Toronto with lower walkability scores, labeled as 'low-low' in Figure 3.

\section{2 - Walk Score and MCDA-based Comparison}

After initial comparisons between SAW and OWA evaluations, their rankings of DAs are compared to Walk Score's evaluation. Figure 4 below shows a heat map of Toronto's Walk Score rankings, created by Walk Score. When visually compared with the SAW and OWA walkability score maps (Figures 1 and 2) it is evident that both evaluations provide similar overall patterns of walkability for Toronto.

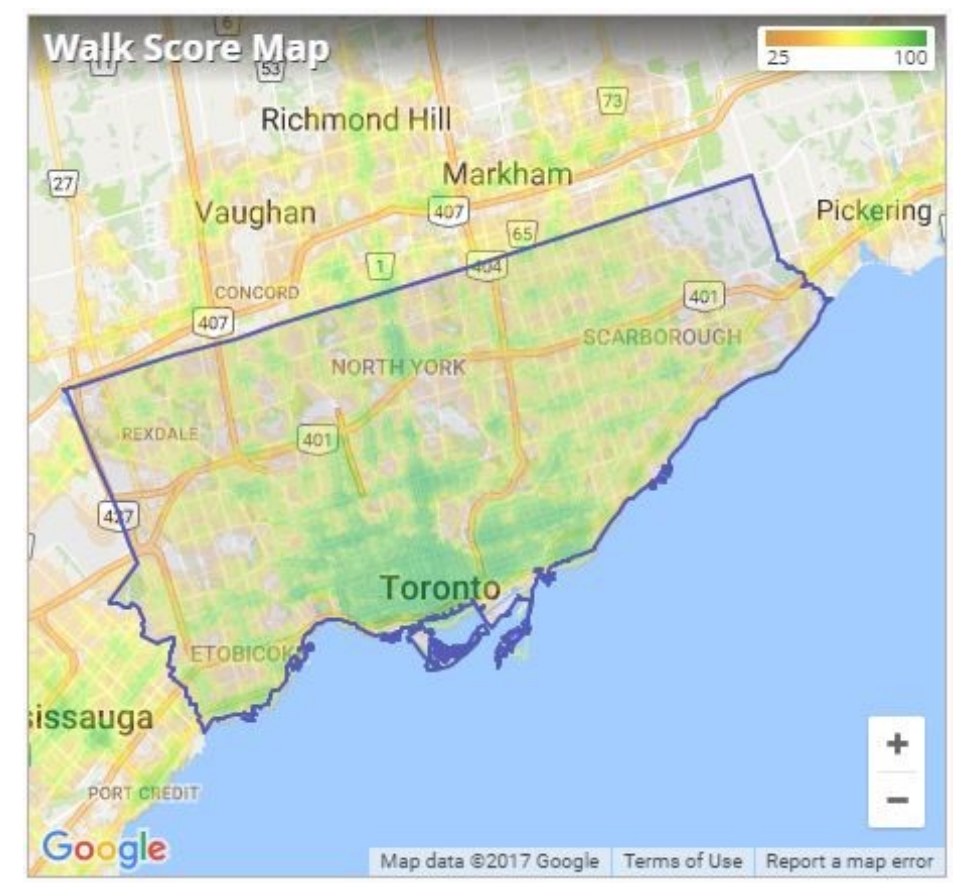

Figure 4 - Heat Map of Walk Score's Evaluation of Toronto's Walkability Source: Walkscore.com

Downtown Toronto has the highest walkability scores and these scores decrease the further away one travels from the downtown core. Additionally, visible in Walk Score's heat map areas within proximity to Yonge Street, from downtown to Toronto's northern border, appear to have higher scores. Both SAW and OWA maps (Figures 1 and 2) show numerous DAs with slightly higher walkability scores closer to Yonge Street within Toronto's boundaries, however this pattern does not exist continuously along the street.

When comparing Walk Score to the MCDA-based results on a smaller scale, there is additional evidence of similarities. Four of the ten neighbourhoods listed as having the highest Walk Scores, seen in Table 11 below, are present within the top 10 SAW and OWA rankings 
(Tables 9 and 10). Six of Walk Score's highest ranking neighbourhoods are not listed as having any of the highest-ranking DAs for SAW and OWA methods. However, it is still evident that these neighbourhoods' DAs have relatively high walkability scores for both MCDA-based methods, as seen in Figures 1 and 2 above.

Table 11 - Walk Score Neighbourhood Ranking of Walkability

\begin{tabular}{r|l|r|r|l|r}
\hline \multicolumn{2}{c|}{ Most Walkable } & \multicolumn{3}{c}{ Least Walkable } & Score \\
\hline Rank & Neighbourhood & Score & Rank & Neighbourhood & 54 \\
\hline 1 & Bay Street Corridor & 99 & 131 & Cliffcrest & 54 \\
2 & Church-Yonge Corridor & 98 & 132 & Highland Creek & 54 \\
3 & Kensington-Chinatown & 97 & 133 & Thistletown-Beaumond Heights & 54 \\
4 & University & 97 & 134 & Centennial Scarborough & 53 \\
5 & Palmerston-Little Italy & 95 & 135 & Morningside & 51 \\
6 & Mount Pleasant West & 95 & 136 & Willowridge-Martingrove-Richview & 49 \\
7 & Moss Park & 95 & 137 & Edenbridge-Humber Valley & 48 \\
8 & Annex & 94 & 138 & Elms-Old Rexdale & 48 \\
9 & Trinitiy-Bellwoods & 94 & 139 & Princess-Rosethorn & 42 \\
10 & North St. James Town & 93 & 140 & Rouge & \\
\hline
\end{tabular}

In contrast to the ten most walkable neighbourhoods, only two of Walk Score's least walkable neighbourhoods were identified as having any of SAW and OWA's ten least walkable DAs. The two neighbourhoods that were present for both Walk Score and MCDA-based methods were Rouge and Morningside. Rouge is identified as the least walkable neighbourhood according to Walk Score. The SAW and OWA methods evaluated one DA within Rouge as the third and fourth least walkable DA respectively. A DA in the Morningside neighbourhood was identified as the least walkable for both SAW and OWA methods, while the neighbourhood was ranked the sixth least walkable in Walk Score's evaluation.

The lack of similarity between rankings is not surprising, as the MCDA-based methods uses different spatial units to those used by Walk Score. Walk Score's ten most and least walkable neighbourhoods contain a large number of DAs, which may not be all represented when observing only the ten most and least walkable DAs from the MCDA-based results. Expanding these tables to include a larger number of DAs may allow us to see a larger number of Walk Score's most and least walkable neighbourhoods included in the study's results. In conclusion, there is a strong correlation between both MCDA-based evaluations and Walk Score for Toronto overall. Each evaluation identified Toronto's downtown core as having the highest 
walkability scores and areas further from downtown as having lower walking scores. To further explore the relationships between scores, DAs within specific neighbourhoods will be analyzed and compared to Walk Score.

\subsection{1 - Niagara "Liberty Village"}

Liberty Village is found within the Niagara neighbourhood just west of downtown Toronto. It is bordered by King Street West to the north, Dufferin Street to the west, the Gardiner Expressway to the south and Strachan Avenue to the east. The Niagara neighbourhood as a whole is bordered by Queen Street West to the north, Dovercourt Road and Atlantic Avenue to the west, the lake shore to the south and Bathurst Street to the west. Liberty Village originated as a prominent industrial area in the late 19th century due to its proximity to the nearby railway. However, by the 1970s, manufacturing operations began declining in the area due to the shift from rail to road shipping of goods. Neglected buildings in the area soon become home for artists looking for studio space (Wieditz, 2007). Local artists began creating organized exhibitions of artwork and founded numerous festivals within the area (Catungal, 2009). The growth of a community in the King and Dufferin area attracted real estate developers and municipal government involvement. Eventually, low income tenants were evicted from buildings in the area, in order for property owners and real estate developers to rebuild the neighbourhood. Property owners began marketing the neighbourhood to new businesses, attracting IT and new-media companies (Catungal, 2009). The gentrification of the area allowed property owners to begin showcasing the neighbourhood as a brand name called 'Liberty Village', bringing in young professionals and promoting its close proximity to downtown Toronto and numerous services and amenities (Wieditz, 2007). When developing Liberty Village, a greater emphasis was placed on pedestrians. Thus, it is beneficial to compare the walkability evaluations of Walk Score and the MCDA-based methods to determine if the urban developers were successful.

In Figures 5 and 6 below, the walkability scores of both SAW and OWA methods are presented. Upon observation, it is evident that a significant portion of the Niagara neighbourhoods' DAs have high walkability scores relative to the city as a whole. The SAW evaluation classified more DAs with lower scores, relative to the OWA evaluation. Two of which are home to a significant population, living in semi-detached homes and apartment 
buildings, and make up a large portion of Liberty Village. In the OWA evaluation, fewer DAs are identified as having lower walkability scores and a smaller portion of Liberty Village is given a lower score. A reason for the differences between MCDA methods is the presence of order weights in the OWA method. The presence of certain walkability factors within the lower scored DAs in the SAW method, may have had higher order weights assigned to them in the OWA method. This would result in these DAs having higher scores in the OWA evaluation than their resulting SAW scores. Although a specific DA within Liberty Village may have a lower walkability score due to lower densities of the walkability factors, these services, and amenities may be present within a short distance in a nearby DA.

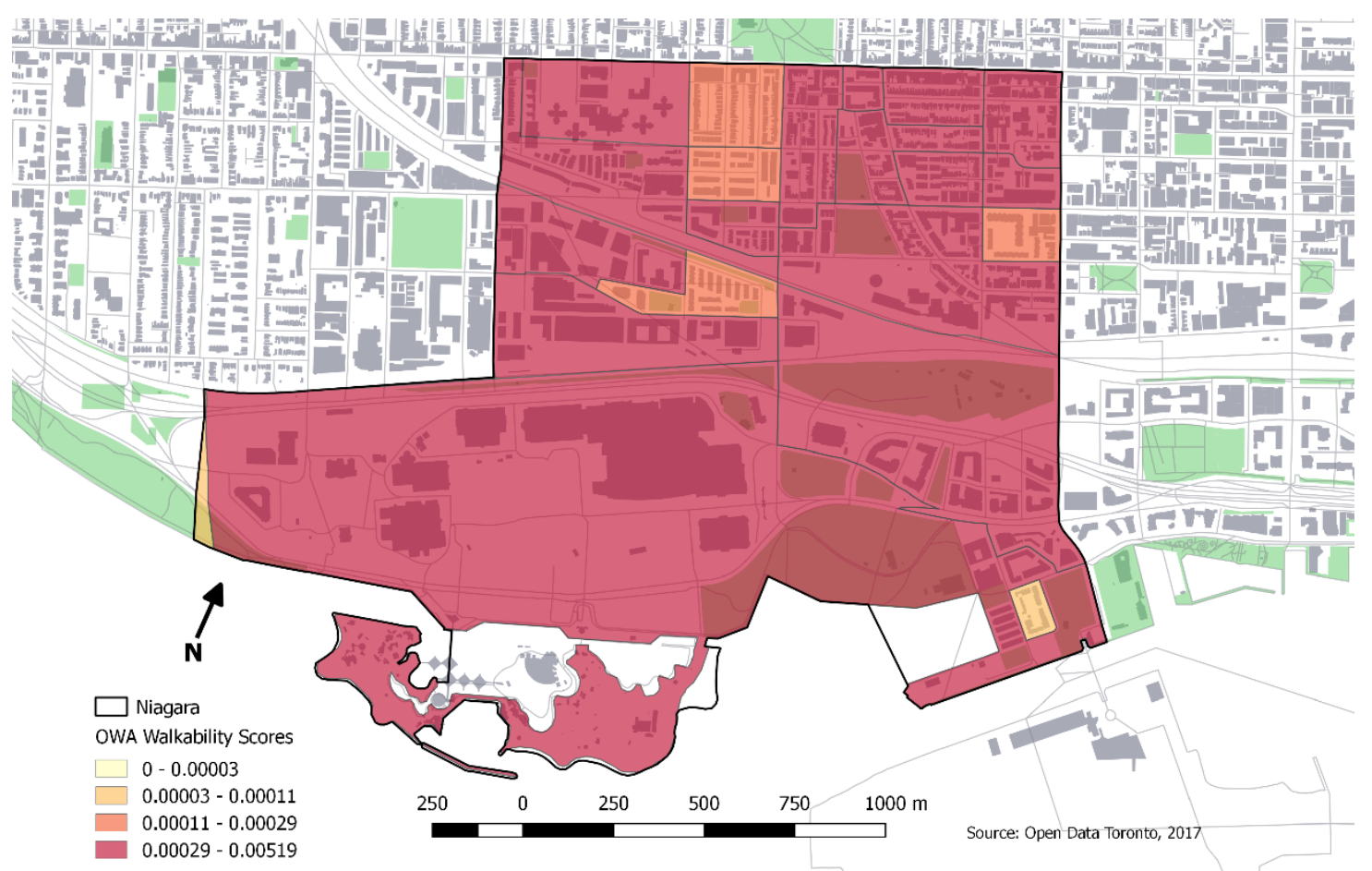

Figure 5 - Niagara Neighbourhood SAW Walkability Scores 


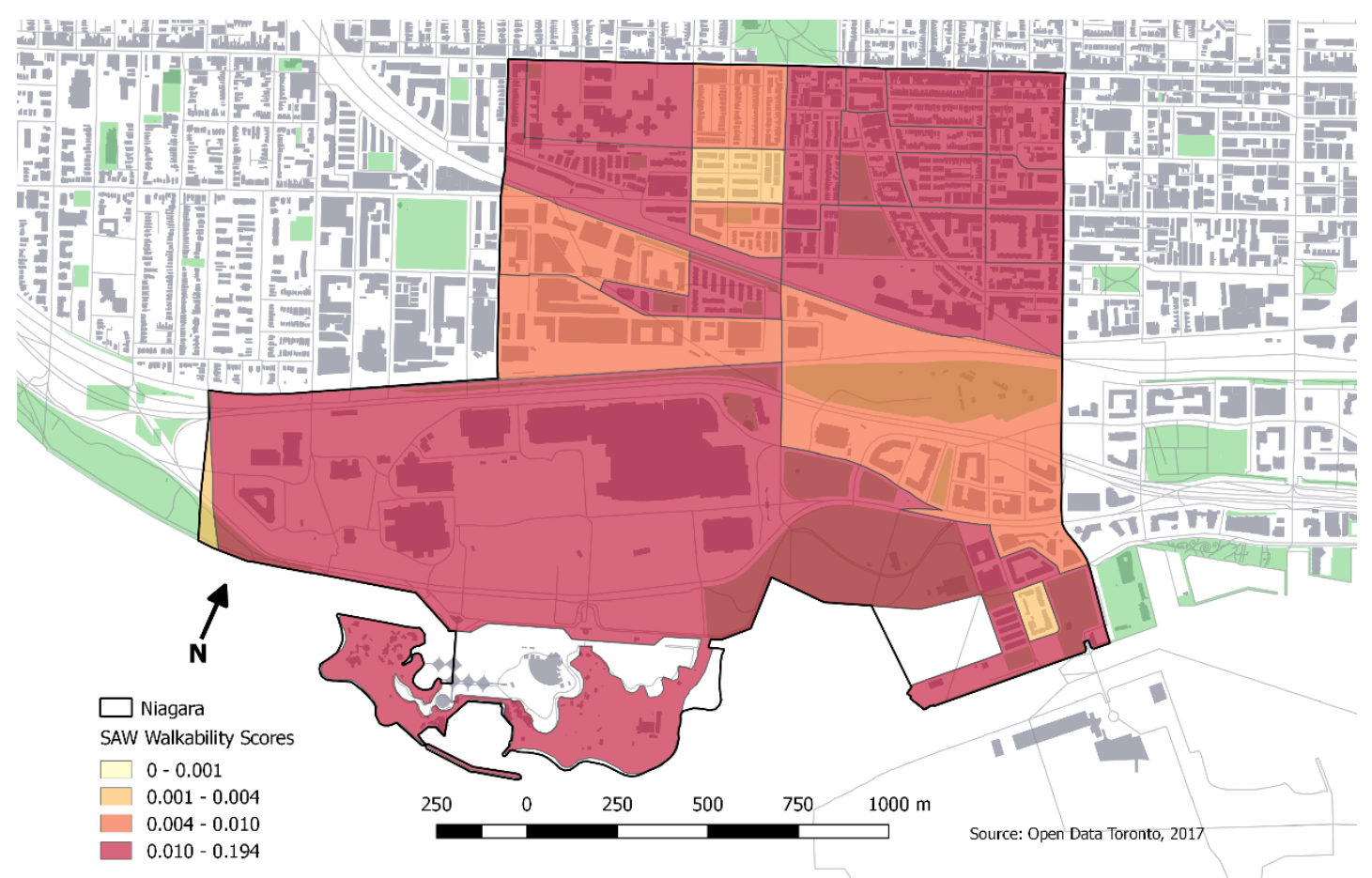

Figure 6 - Niagara Neighbourhood OWA Walkability Scores

Walk Score's methodology, which does not limit scores by specific geographic boundaries, supports this notion. Thus, when calculating walkability scores for Liberty Village, services and amenities in nearby areas were accounted for. This is evident in Walk Score giving Liberty Village a walkability score of 92 out of 100 and identifying the area as a Walker's Paradise (Walk Score, 2017). Walk Score gave the Niagara neighbourhood an overall score of 84, ranked $32^{\text {nd }}$ of 140 Toronto neighbourhoods. In conclusion, when observing the neighbourhood as a whole, both MCDA-based methods and Walk Score provide similar evaluations of the neighborhood's walkability. With the support of both Walk Score and the MCDA-based methods, it appears that efforts to increase the walkability of Liberty Village and Niagara as a whole have been successful.

\subsection{2 - The Beaches}

The Beaches are located east of downtown Toronto along the waterfront. Its boundaries include Victoria Park Avenue to the east, Kingston Road to the north, Coxwell Avenue to the west and Lake Ontario to the south. The area originally gained popularity as a summer resort (Luka, 2006). The extension of Queen Street and its streetcar line, eastward through the area caused an exponential growth in developments, including cottage homes and amusement parks (Luka, 
2006). The last operational amusement park closed in 1925 (Toronto Neighbourhood Guide, 2017). After this time, more year-round residential homes were constructed in the neighbourhood. Today, the population of the area continues to grow exponentially and attracts people from around Toronto to its numerous services and amenities, in addition to its various festivals. Unlike Liberty Village, the Beaches was not initially developed as a pedestrianoriented neighbourhood. However, in recent years there has been a shift in the area from focusing on vehicles to pedestrians (Golovkin, 2016). Measuring the walkability of this neighbourhood determines if any further improvements are necessary.

Walk Score has labeled The Beaches as the 24th most walkable neighbourhood in Toronto with a score of 88 out of 100, stating that most errands can be accomplished by foot and the neighbourhood has excellent public transit (Walk Score, 2017). This high walkability score is visible in a majority of DAs created by the MCDA-based evaluations, as seen in Figures 7 and 8 below.

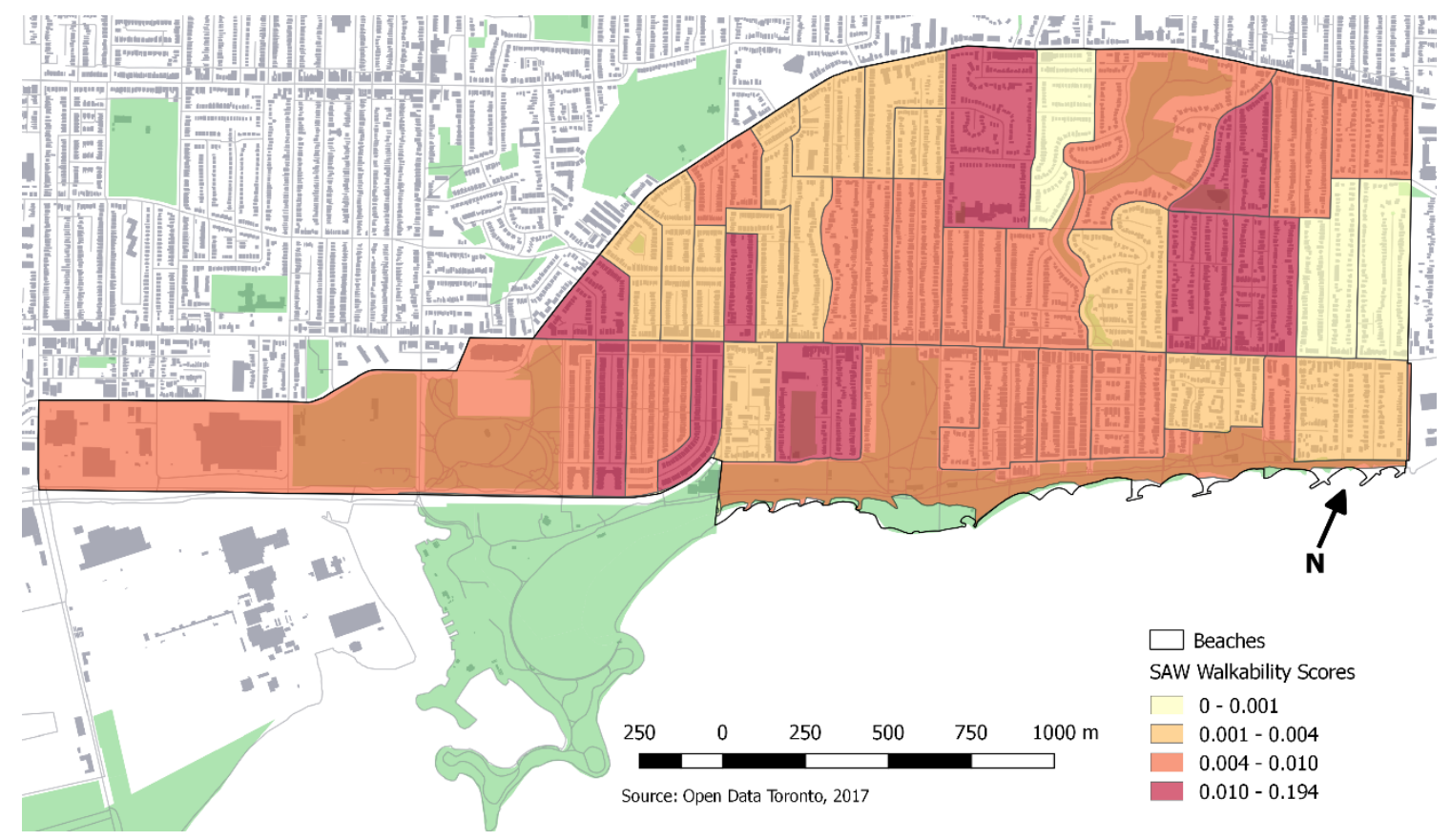

Figure 7 - The Beaches Neighbourhood SAW Walkability Scores 


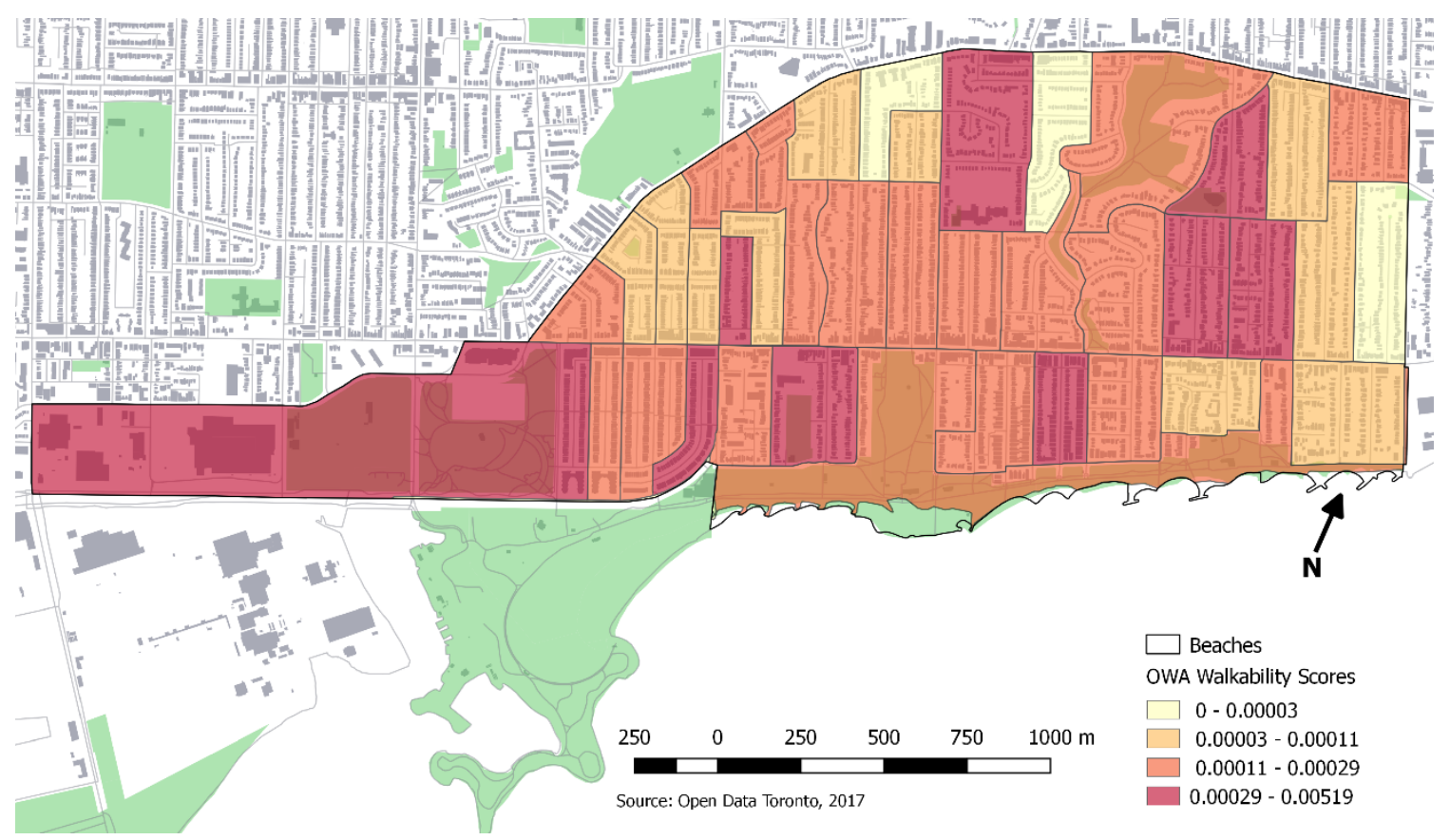

Figure 8 - The Beaches Neighbourhood OWA Walkability Scores

Upon initial observations of the Beaches as an entity, both SAW and OWA methods created similar evaluations of the neighbourhood DAs. Many of the DAs have similar scores relative to other areas of the city, based on the colour scheme in the resulting maps. Although a majority of the neighbourhood has high walkability scores, there are still numerous DAs with low scores. A reason for the lower rankings provided by the MCDA-based methods may be due to the concentration of services and amenities found along Queen Street East, while a majority of the rest of the neighbourhood is residential housing. The geographic boundaries of DAs which include part of Queen Street East only include a small portion of the street's services and amenities, thus causing the density of those walkability factors within each DA to be limited. Additionally, DAs north of Queen Street East are predominantly older detached and semidetached homes, thus reducing the overall population density of these DAs. Areas south of Queen Street East are newer developments, thus are designed to home a higher density of families. Lastly, a limited number of intersections are present within the neighbourhood overall as it was originally designed as a vehicle-oriented area.

In conclusion, these limitations on the study's criteria have a negative impact on the resulting walkability scores created by both SAW and OWA methods. However, there are noticeable changes that has increased the walkability of certain areas within the Beaches. Areas 
of improvement are still visible in the MCDA evaluations when observing the neighbourhood on a DA-scale. The differences in methodology between Walk Score, SAW and OWA methods plays a key role in the discrepancies found in walkability scores, however each evaluation has created consistent scores for the neighbourhood.

\subsection{4 - Rouge}

The Rouge neighbourhood is located in the northeastern part of Toronto, formerly a part of Scarborough. It is bordered by Steeles Avenue to the North, the Toronto-Pickering town line to the East, highway 401 to the South and Markham Road and Morningside Avenue to the West. Rouge originated as rural farmland until the 1950s when highway 401 was extended eastward through the area (Toronto Neighbourhood Guide, 2017). This extension allowed developers to begin urbanizing the area with predominantly residential subdivisions. The landscape of the Rouge neighbourhood has not changed significantly since its initial urbanization, unlike Niagara and the Beaches.

Walk Score identified Rouge as the least walkable neighbourhood in Toronto, ranked 140th overall, with a walk score of 42 out of 100 . Walk Score states that most errands can only be accomplished with the use of a vehicle and that the area has good public transit (Walk Score, 2017). This ranking is reflected in both SAW and OWA evaluations as seen in Figures 9 and 10 below. 


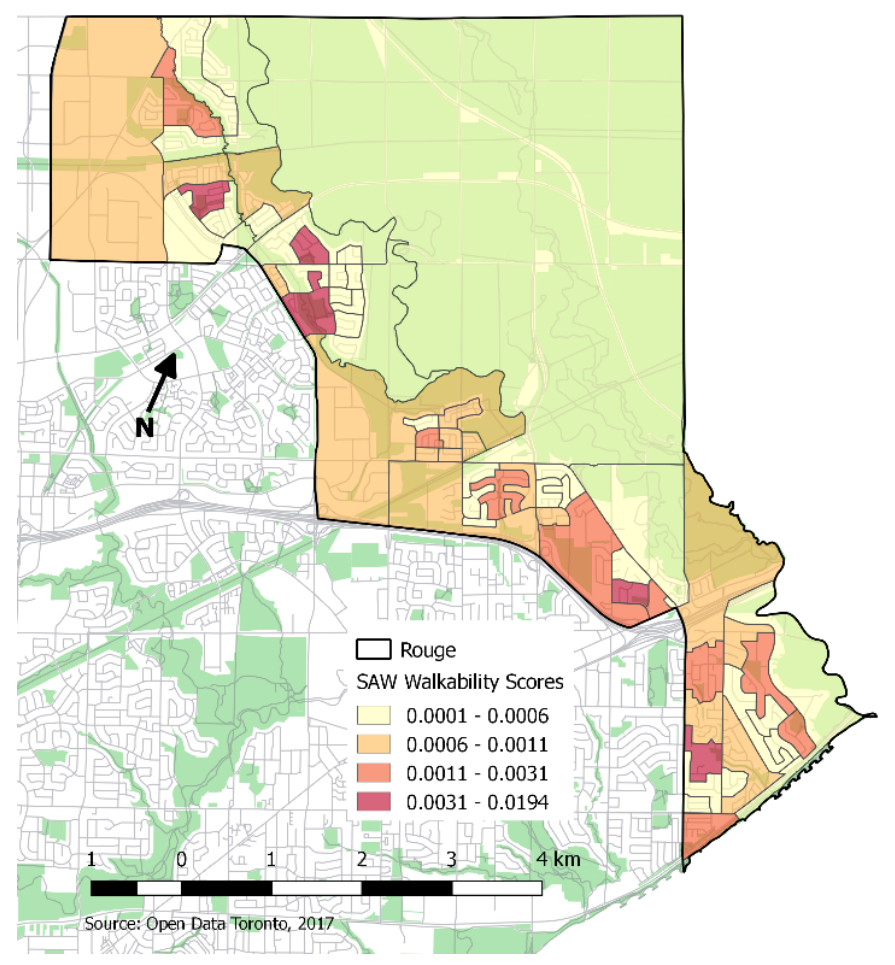

Figure 9 - Rouge Neighbourhood SAW Walkability Scores

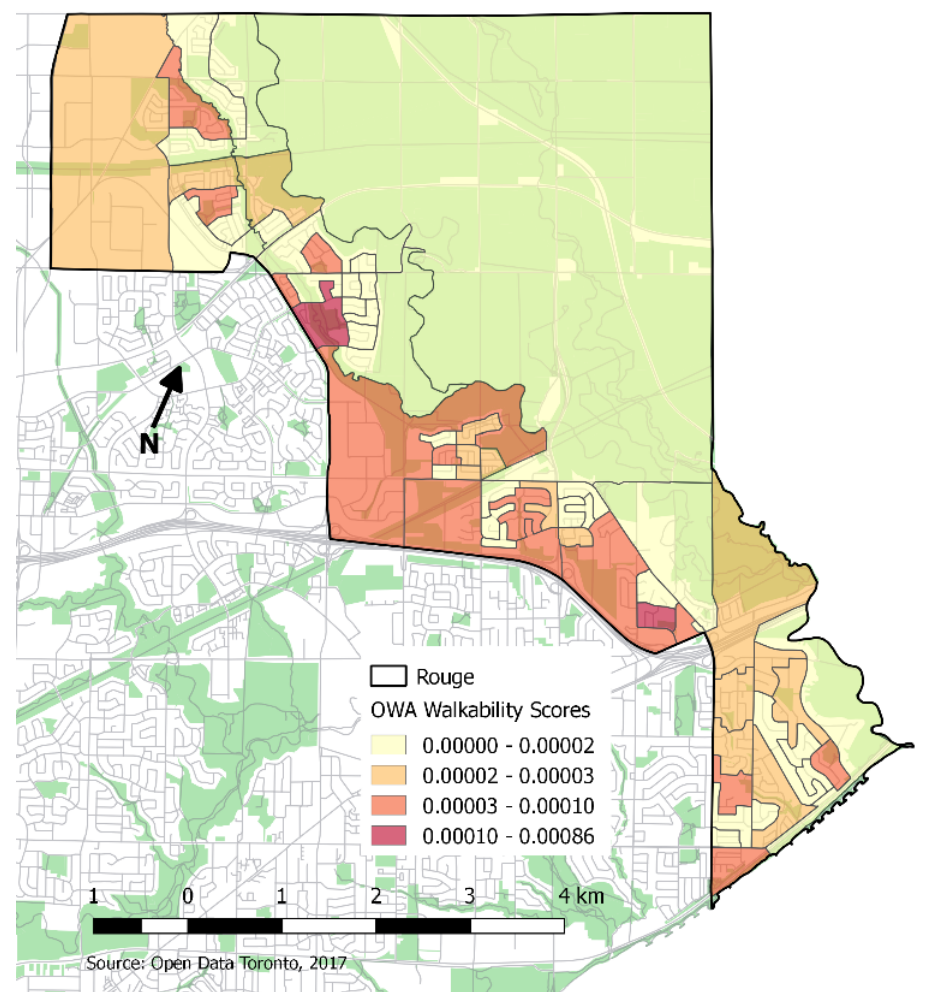

Figure 10 - Rouge Neighbourhood OWA Walkability Scores 
For both SAW and OWA evaluations, most DAs have been given low scores relative to the rest of the city. Services and amenities within the area are often clustered together within plazas located on major arterial roads. Thus, only a few DAs within the neighbourhood are given higher scores due to a higher density of services and amenities in the area. A significant portion of the neighbourhood is green space including forested areas and farm land. The large amount of green space greatly reduces the population density of the neighbourhood thus limiting the amount of services and amenities the city may deem necessary for the area. Unlike many other neighbourhoods, Rouge is predominantly a residential area with a focus on the presence of schools rather than necessary services and points of interest. Thus, in order to fulfill any daily errands, residents must drive a significant distance. This creates challenges for those without a vehicle who live in the area. In addition, walking to nearby neighbourhoods creates dangerous environments for pedestrians as they must travel significant distances and walk over major highways and arterial roads.

In conclusion, it is evident that the severe lack of the walkability criteria in the Rouge neighbourhood has caused both Walk Score and the MCDA-based methods to classify the area as one of, if not the least walkable neighbourhood in Toronto. 


\section{6 - Discussion and Conclusion}

\section{1 - Limitations and Future Research}

When comparing the resulting walkability evaluations of Toronto DAs and neighbourhoods, it is important to note that the scores are dependent on the methodology used. A limitation of this study is the inability to compare the raw resulting scores of Walk Score and the MCDA-based methods, SAW and OWA. The resulting scores of Walk Score and the MCDA-based methods both lacked units of measurement, thus could not be objectively compared. Therefore, the scores were compared by ranking the DAs. Further analysis could examine the use of non-linear standardization when normalizing criteria values prior to adding the criterion weights. This study assumed that Walk Score's evaluations were normally distributed, thus a linear standardization of the criteria values was performed. Future research may benefit from recognizing the "natural" skewness of Walk Score's evaluations to give urban neighbourhoods higher scores.

Additional limitations include the significant differences in methodology used by Walk Score and the MCDA-based methods. Walk Score's evaluation emphasized the proximity of services and amenities to a point and were not limited to searching for the criteria within a fixed geographic boundary. However, when using MCDA-based methods, DAs were used as fixed boundaries to determine the walkability at a larger scale. Thus, the presence of criteria in a nearby DA would not be accounted for when determining the walkability of each DA. In order to address this issue, future research could examine the relationship between walkability scores calculated using varying boundary scales, such as DAs compared to neighbourhood boundaries.

Lastly, criterion weights used when calculating SAW and OWA walkability scores are highly subjective. Weights for each criterion were determined based on a pairwise comparison technique, determining how much more important one criterion is over another. These decisions are subjective to the decision-maker(s) and their preferences. Thus, the resulting walkability scores of Toronto DAs would differ depending on who is making the decisions. For this study, only a single decision-maker's preference was used to determine the criterion weights. In future studies, the use of a group of decision-makers' with interest in neighbourhood walkability would be beneficial to reduce the presence of bias results. 


\section{2 - Summary and Conclusion}

In this study, MCDA techniques, specifically SAW and OWA methods, were used to measure the walkability of Toronto DAs. This was done to compare the resulting walkability evaluations to Walk Score, a popular urban accessibility index, and determine if MCDA methods could be used for further walkability evaluations. Similar criteria used by Walk Score was selected for this study, representing all indicators of walkability. Criterion weights were created using the pairwise comparison matrix, determining the importance of each criterion compared to one another. These weights are subjective to the preferences of the decision maker involved in the study. Criteria values were determined by calculating the density of each criterion within each DA in the city of Toronto. The resulting values were then standardized using the score range transformation procedure, ranking the values from 0 to 1 . For the SAW method, criterion weights were multiplied by their associated criteria values. The resulting weighted criteria values were summed for each dissemination area.

For the OWA method, the weighted criteria values were rearranged in descending order for each DA. Order weights were determined by the number of criteria involved in the study and the amount of tradeoff wanted by the decision maker. The first order weight was then multiplied by the highest weighted criterion value within each DA, followed by the second order weight multiplied by the second highest weighted criterion value. This process repeats for the remaining number of criterion. The resulting ordered and weighted criteria values were summed for each dissemination area. The resulting scores for both SAW and OWA methods represent the walkability of each DA. The area with the highest score is deemed the most walkable.

The resulting scores for both SAW and OWA methods identified similar areas as the most and least walkable. These DAs and their associated neighbourhoods were compared to Walk Score's most and least walkable neighbourhoods. Both Walk Score and the MCDA-based methods identified similar areas of the city as the most walkable, predominantly areas located in Toronto's downtown core, when compared visually and using statistical methods. The further a DA is from the downtown core, the lower its associating walkability score. When comparing the least walkable areas of the city, there was a limited correlation between Walk Score and the MCDA-based results. This was predominantly due to the large number of DAs that had a lower score, thus larger number of neighbourhoods could be identified as the least walkable. 
The relationship between Walk Score, OWA and SAW evaluations were observed for three Toronto neighbourhoods, Niagara, the Beaches and Rouge. Niagara and the Beaches were chosen for this study due to their shift in focus from vehicles to pedestrian-oriented environments. However, both neighbourhoods began this shift in different ways. It was evident when comparing Walk Score and the MCDA-based evaluations for Niagara, that both methods acknowledged the presence of walkability indicators currently situated in the neighbourhood, as each evaluation gave the neighbourhood a high walkability score. This was somewhat true for the Beaches as well, as Walk Score gave the neighbourhood a high walkability score and the resulting MCDA-based walkability scores were high, compared to the city as a whole, however certain areas within the neighbourhood had lower scores. Lastly, Rouge was also observed as it was deemed one of the least walkable neighbourhoods in Toronto in every evaluation. When observing the pattern of the walkability scores at a DA level, it was evident that the MCDAbased methods gave the DAs similar rankings as Walk Score.

Overall, the use of small geographic boundaries when performing MCDA limits the walkability scores, due to the limited number of services and amenities that could exist within each DA. However, comparing each evaluation's ranking of DAs and neighbourhoods, the most and least walkable areas of Toronto were identifiable using both MCDA-based methods and were similar to Walk Score's results when observing the city as a whole. 


\section{References}

Afshari, Alireza, Majid Mojahed and Mohd Rosnah. "Simple Additive Weighting approach to Personnel Selection problem." International Journal of Innovation, Management and Technology 1.5 (2010). Web.

Bellman, R. E. and L. A. Zadeh. "Decision-making in a fuzzy environment." Management Science 17.4 (1970): 141-164. Web.

Bhat, Chandra, et al. Urban Accessibility Index: Literature Review. Austin: Texas Department of Transportation, 2000. Web.

Bourne, Larry S. and David Ley. The Changing Social Geography of Canadian Cities. Montreal: McGill-Queens U Press, 1993. Print.

Canada, Statistics. Census Dictionary - Dissemination Area (DA). 27 November 2015. Web. 31 July 2017.

Carr, Lucas J., Shira I Dunsiger and Bess H. Marcus. "Validation of Walk Score for Estimating Access to Walkable Amenities." British Journal of Sports Medicine 45.14 (2011). Web.

Carr, Lucas J., Shira I. Dunsiger and Bess H. Marcus. "Walk Score TM as a global estimate of neighbourhood walkability." Am J. Prev. Med. (2010): 460-463. Web.

Catungal, John Paul, Deborah Leslie and Yvonne Hii. "Geographies of Displacement in the Creative City: The Case of Liberty Village, Toronto." Urban Studies (2009): 1095 1114. Web.

De Cambra, Paulo Jorge Montiro. "Pedestrian Accessibility and Attractiveness Indicators for Walkability Assessment." Urbanismo e Ordenamento do Territorio (2012). Web.

Drobne, Samo and Anka Lisec. "Multi-attribute Decision Analysis in GIS: Weighted Linear Combination and Ordered Weighted Averaging." Informatica 33 (2009): 459-74. Web.

Duncan, Dustin T., et al. "Validation of Walk Score for Estimating Neighbourhood Walkability: an Analysis of Four US Metropolitan Areas." International Journal of Environmental Research and Public Health 8.12 (2011): 4160-179. Web.

_. "Validation of Walk Scores and Transit Scores for estimating neighborhood walkability and transit availability: a small-area analysis." GeoJournal 78.407 (2013).

Evans, G. "Accessibility, Urban Design and the Whole Journey Environment." Built Environment 35.3 (2009): 366-385. Web.

Feick, Robert and Brent Hall. "A method for examining the spatial dimension of multi-criteria weight sensitivity." International Journal of Geographic Information Science 18.8 (2004): 815-40. Web.

Forsyth, Ann and Michael Southworth. "Cities Afoot - Pedestrians, Walkability and Urban Design." Journal of Urban Design 13.1 (2008): 1-3. Web.

Grasser, G., et al. "Objectively Measured Walkability and Active Transport and Weight-Related Outcomes in Adults: A Systematic Review." International Journal of Public Health 58.4 (2013): 615-25. Web.

Guide, Toronto Neighbourhood. The Beaches. 2017. Web. 2017. 
Halstead, J.M. and S.C. Deller. "Public Infrastructure in Economic Development and Growth: Evidence from Rural Manufacturers." Journal of the Community Development Society 28.2 (1997): 149-69. Web.

Hess, Paul Mitchell and Jane Farrow. "Walkability in Toronto's high-rise neighbourhoods: Final Report." Canadian Electronic Library (2014). Web.

Kickert, WJM. "Fuzzy theories of decision making: A critical review." Springer Science \& Business Media 3 (1979). Web.

Knox, P.L. "The intraurban Ecology of Primary Medical Care: Patterns of Accessibility and their Policy Implications." Environment and Planning (1978): 415-435. Web.

Larsen, Kristian, et al. "The Influence of the Physical Environment and Sociodemographic Characteristics on Children's Mode of Travel to and from School." American Journal of Public Health 99.3 (2009): 520-26. Web.

Malczewski, Jacek and Claus Rinner. "Exploring multicriteria decision strategies in GIS with linguistic quantifiers: A case study of residential quality evaluation." Journal of Geographical Systems 7.2 (2005): 249-68. Web.

Malczewski, Jacek. GIS and Multicriteria Decision Analysis. New York: J. Wiley \& Sons, 1999. Print.

Munda, G. "Multicriteria evaluation in a fuzzy environment: theory and applications in ecological economics." Theory and Applications in Ecological Economics (1995). Web.

Nykiforuk, Candace I.J., et al. "Check the Score: Field Validation of Street Smart Walk Score in Alberta, Canada." Preventitive Medicine Reports (2016): 532-539. Web.

Pirie, G.H. "Measuring Accessibility: A Review and Proposal." Environment and Planning (1979): 299-312. Web.

Rinner, Claus and Jacek Malczewski. "Web-enabled spatial decision analysis using Ordered Weighted Averaging (OWA)." Journal of Geographical Systems 4 (2002): 385-403. Print.

Rogers, Shannon H., et al. "Examining Walkability and Social Capital as Indicators of Quality of Life at the Municipal and Neighbourhood Scales." Applied Research in Quality of Life 6.2 (2011): 201-213. Web.

Roy, Bernard. Multicriteria Methodology for Decision Aiding. Springer US, 1996. Web.

Saaty, T.L. The Analytic Hierarchy Process. New York: McGraw-Hill, 1980. Web.

Statistics Canada. Definition - Dissemination Areas. 2017. Web. 2017.

Toronto Neighbourhood Guide. Rouge. 2017. Web. 2017.

—. The Beaches. 2017. Web. 2017.

Walk Score. Living in Niagara Toronto. 2017. Web. 2017.

—. Living in Rouge Toronto. 2017. Web. 2017.

-. Living in the Beaches Toronto. 2017. Web. 2017.

—. Walk Score Methodology. 2017. Web. 2017.

Wieditz, Thorben. "Liberty Village: The Makeover of Toronto's King and Dufferin Area." Centre for Urban and Community Studies (2007). Web. 
World Health Organization. "Global Health Observatory: Prevalence of Insufficient Physical Activity." Manual. 2012. Web.

Yager, R.R. "On ordered weighted averaging aggregation operators in multi-criteria decision making." IEEE Transactions on Systems, Man and Cybernetics 18.1 (1988): 183-190. Web.

Zopounidis, Constantin and Michael Doumpos. "Multicriteria classification and sorting methods: A literature review." European Journal of Operational Research (2002): 229-246. Web. 